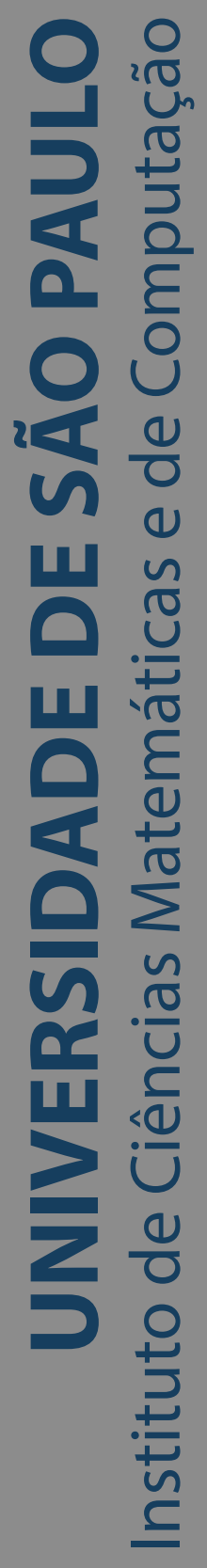

Subvariedades de codimensão 2 em formas espaciais

\title{
Cleidinaldo Aguiar Souza
}

Tese de Doutorado do Programa de Pós-Graduação em Matemática (PPG-Mat) 

Assinatura:

\title{
Cleidinaldo Aguiar Souza
}

Subvariedades de codimensão 2 em formas espaciais

\begin{abstract}
Tese apresentada ao Instituto de Ciências Matemáticas e de Computação - ICMC-USP, como parte dos requisitos para obtenção do título de Doutor em Ciências - Matemática. VERSÃO REVISADA
\end{abstract}

Área de Concentração: Matemática

Orientador: Prof. Dr. Fernando Manfio 
Ficha catalográfica elaborada pela Biblioteca Prof. Achille Bassi e Seção Técnica de Informática, ICMC/USP, com os dados inseridos pelo(a) autor(a)

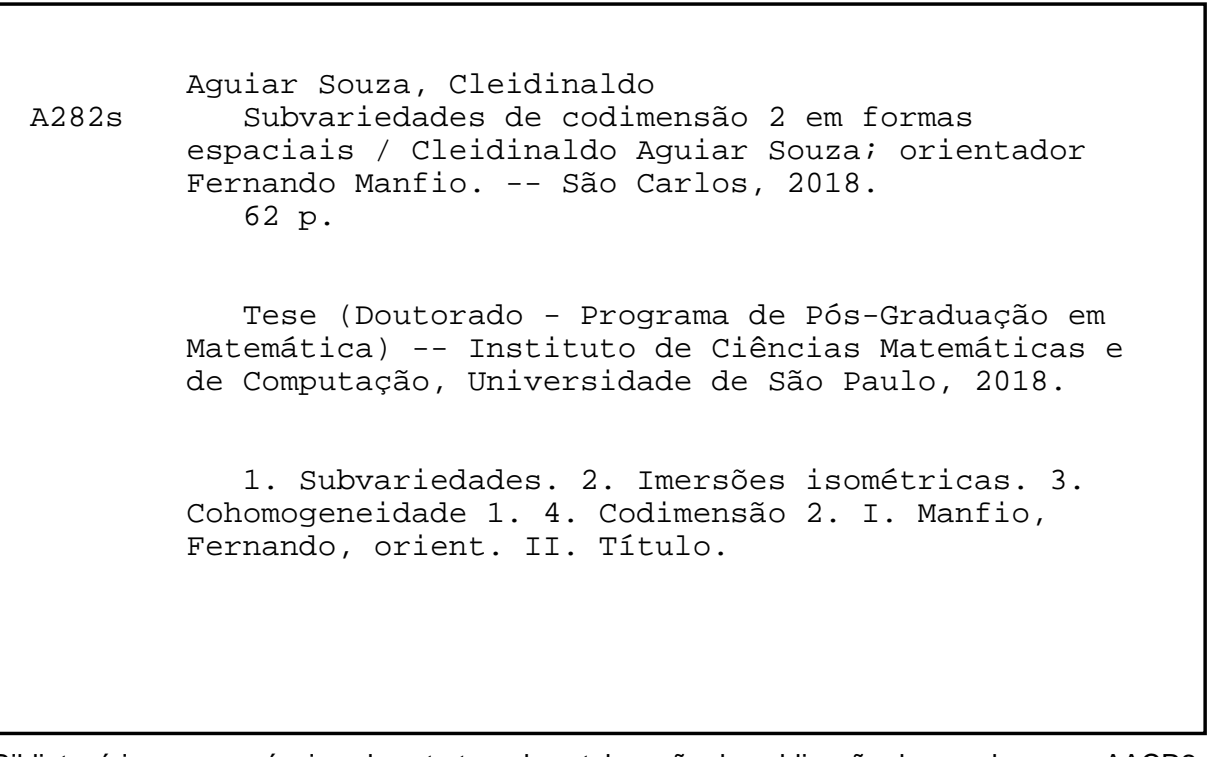

Bibliotecários responsáveis pela estrutura de catalogação da publicação de acordo com a AACR2: Gláucia Maria Saia Cristianini - CRB - 8/4938

Juliana de Souza Moraes - CRB - 8/6176 
Cleidinaldo Aguiar Souza

\section{Submanifolds of codimension 2 into space forms}

Doctoral dissertation submitted to the Institute of Mathematics and Computer Sciences - ICMC-USP, in partial fulfillment of the requirements for the degree of the Doctorate Program in Mathematics. FINAL VERSION

Concentration Area: Mathematics

Advisor: Prof. Dr. Fernando Manfio

\section{USP - São Carlos}

July 2018 

À minha mãe, Ednalva Maria Aguiar Souza (in memorian). 

Agradeço a Deus, por tudo.

Agradeço aos meus pais: Ednalva e Raimundo Leal, pelas palavras sábias.

Agradeço aos meus irmãos: Ligia, João Paulo, Larisse, Antônio Raelson, pelo apoio nos momentos mais difíceis.

Agradeço a minha namorada, Arianne Kelly, minha companheira inseparável.

Agradeço aos amigos que conheci em São Carlos: Joás pelas conversas sobre matemática e sobre a vida, Rafaela e Carol por boas risadas, Alexandre e Gregory pelo convívio.

Agradeço ao meu orientador, Fernando Manfio, por acreditar em mim e me apresentar um problema tão interessante.

Agradeço ao departamento de matemática da UFPI. 

"As invenções são, sobretudo, o resultado de um trabalho de teimoso."

(Santos Dumont) 



\section{RESUMO}

SOUZA, C. A. Subvariedades de codimensão 2 em formas espaciais. 2018. 62 p. Tese (Doutorado em Ciências - Matemática) - Instituto de Ciências Matemáticas e de Computação, Universidade de São Paulo, São Carlos - SP, 2018.

Um problema central em teoria de subvariedades é estudar imersões isométricas $f: M^{n} \rightarrow \mathbb{Q}_{c}^{n+k}$ de uma variedade Riemanniana completa em uma forma espacial sob a ação de um subgrupo conexo e fechado do grupo de isometrias $I s o(M)$. Esse estudo teve início com o relevante trabalho de Kobayashi (KOBAYASHI, 1958), que provou que se $M^{n}$ é uma hipersuperfície compacta e homogênea no espaço Euclidiano, então $M^{n}$ é isométrica à esfera usual.

Neste trabalho estudamos imersões isométricas em formas espaciais com codimensão igual a 2. Mais precisamente, obtemos uma classificação das imersões isométricas $f: M^{n} \rightarrow \mathbb{Q}_{c}^{n+2}$ de uma variedade Riemanniana completa sob a ação de cohomogeneidade 1 de um subgrupo fechado $G \subset I s o(M)$, de modo que as órbitas principais são hipersuperfícies umbílicas de $M$.

Palavras-chave: Subvariedades; Imersões Isométricas; Cohomogeneidade 1; Codimensão 2. 



\section{ABSTRACT}

SOUZA, C. A. Submanifolds of codimension 2 into space forms. 2018. 62 p. Tese (Doutorado em Ciências - Matemática) - Instituto de Ciências Matemáticas e de Computação, Universidade de São Paulo, São Carlos - SP, 2018.

An important problem in submanifold theory is to study isometric immersions $f: M^{n} \rightarrow \mathbb{Q}_{c}^{n+k}$ into a space form of a complete Riemannian manifold of dimension $n$ acted on by a closed connected subgroup of its isometry group $I s o(M)$. This study was initiated by Kobayashi (KOBAYASHI, 1958), who proved that if $M^{n}$ is a compact and homogeneous hypersurface into Euclidean space, then $M^{n}$ must be a round sphere.

In this work we study isometric immersions into a space form with codimension 2. More precisely, we give a complete classification of isometric immersions $f: M^{n} \rightarrow \mathbb{Q}_{c}^{n+2}$ of a complete Riemannian manifold into a space form acted on by a closed connected subgroup $G \subset I s o(M)$ of cohomogeneity one, under the assumption that all principal orbits are umbilical hypersurfaces of $M^{n}$.

Keywords: Submanifolds; Isometric Immersions; Cohomogeneity one; Codimension 2. 



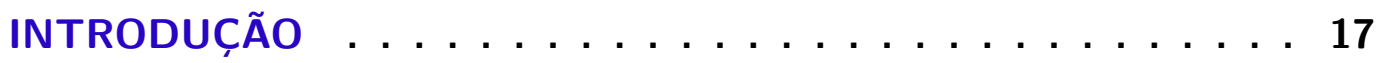

2 SUBVARIEDADES E IMERSÕES ISOMÉTRICAS . . . . . . . . 23

2.1 Subvariedades . . . . . . . . . . . . . . . 23

$2.2 \quad$ Ações de grupos de Lie . . . . . . . . . . . . . . . . 28

$2.3 \quad$ Produto warped de variedades Riemannianas . . . . . . . . . . . . 33

$2.4 \quad$ Produtos warped de imersões isométricas . . . . . . . . . . . . 36

2.5 Teorema de classificação de Dajczer-Tojeiro . . . . . . . . . . . 39

3 SUBVARIEDADES DE CODIMENSÃO DOIS EM FORMAS ESPACIAIS ....................... . . . 43

$3.1 \quad$ Resultados auxiliares .................. 43

$3.2 \quad$ Resultado local . . . . . . . . . . . . . . . . . 46

3.3 Subvariedades Euclidianas de codimensão $2 \ldots \ldots$. . . . . . 50

3.3.1 $\quad 0$ caso compacto . . . . . . . . . . . . . . . . 50

3.3.2 O caso completo . . . . . . . . . . . . . . . . 52

$3.4 \quad$ Subvariedades de codimensão 2 na esfera . . . . . . . . . . . . . 54

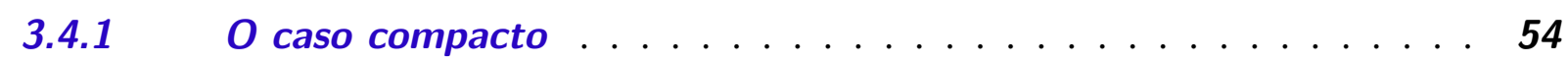

$3.4 .2 \quad$ O caso completo . . . . . . . . . . . . . . . . 55

3.5 Subvariedades de codimensão 2 no espaço hiperbólico . . . . . . 57

3.5.1 O caso compacto . . . . . . . . . . . . . . . 57

$3.5 .2 \quad 0$ caso completo . . . . . . . . . . . . . . . 59

REFERÊNCIAS ........................... 61 



\section{1}

\section{INTRODUÇÃO}

Um problema clássico em teoria de subvariedades é o estudo de imersões isométricas $f: M^{n} \rightarrow \mathbb{R}^{n+k}$ de uma variedade Riemanniana completa e conexa sob a ação de um subgrupo fechado e conexo do grupo das isometrias $I s o(M)$. O primeiro trabalho nessa direção é devido a Kobayashi (KOBAYASHI, 1958), que provou que se $k=1$ e $M^{n}$ é compacta e homogênea, i.e., Iso $(M)$ age transitivamente em $M$, então $f(M)$ é isométrica à esfera usual.

O trabalho de Kobayashi foi generalizado em várias direções. Por exemplo, o caso não compacto é devido a Nagano-Takahashi (NAGANO; TAKAHASHI, 1960) e Harle (HARLE, 1971), que provaram que se $M^{n}$ é homogênea e conexa, então $M^{n}$ é isométrica ao produto $\mathbb{S}^{k} \times \mathbb{R}^{n-k}$. Posteriormente, Ros (ROS, 1988) provou que qualquer hipersuperfície compacta de curvatura escalar constante é isométrica à esfera. As hipersuperfícies homogêneas nas demais formas espaciais foram estudadas por Takahashi (TAKAHASHI, 1971), (TAKAHASHI, 1970), no caso hiperbólico, e por Hsiang (HSIANG; JR, 1971) e Uchida (UCHIDA, 1980), no caso da esfera.

Posteriormente, Podestà-Spiro (PODESTÀ; SPIRO, 1995) provaram que uma hipersuperfície Euclidiana compacta, sob a ação de um subgrupo fechado de seu grupo de isometrias, é rotacional se, e somente se, as órbitas principais são hipersuperfícies umbílicas de $M$. O caso em que a hipersuperfície $M$ é completa e não compacta foi abordado por Mercuri-Seixas (MERCURI; SEIXAS, 2004), que provaram que se $M$ é rígida no infinito, então $M$ é rotacional.

Em (MERCURI et al., 2006), Mercuri-Podestà-Seixas-Tojeiro estudaram hipersuperfícies completas $f: M^{n} \rightarrow \mathbb{R}^{n+1}$, sob a ação de cohomogeneidade 1 de um subgrupo conexo e compacto $G \subset I s o(M)$. Mais precisamente, os autores obtiveram uma classificação completa de tais hipersuperfícies se $n \geq 3$ e $M$ compacta, ou se $n \geq 5$ e as componentes conexas da parte flat de $M$ são limitadas. Em (LIMA, 2002), Lima classificou as hipersuperfícies de cohomogeneidade 1 com óbitas principais umbílicas na esfera Euclidiana. A classificação das hipersuperfícies de cohomogeneidade 1, com órbitas principais umbílicas, no espaço hiperbólico é devido a 
Asperti-Caputi (ASPERTI; CAPUTI, 2003).

O caso de codimensão 2 teve início com o trabalho de Castro-Noronha (CASTRO; NORONHA, 1999), e melhorado posteriormente em (CASTRO; NORONHA, 2002). Basicamente, os autores estudaram subvariedades homogêneas do espaço Euclidiano de codimensão 2, com índice de nulidade relativo $v \leq n-5$ provando, neste caso, que $M^{n}$ é um produto $N^{k} \times \mathbb{R}^{n-k}$, onde $N^{k}$ é isométrica ou a uma esfera, ou a uma hipersuperfície isoparamétrica compacta da esfera, ou é recoberta por $\mathbb{S}^{k-1} \times \mathbb{R}$.

Nesta tese estudaremos imersões isométricas $f: M^{n} \rightarrow \mathbb{Q}_{c}^{n+2}$ de uma variedade Riemanniana $M^{n}$, sob a ação de cohomogeneidade 1 de um subgrupo de Lie conexo e fechado $G \subset I s o(M)$, cujas órbitas principais são hipersuperfícies umbílicas de $M^{n}$. Apresentaremos uma classificação completa de tais subvariedades, tanto para o caso em que $M$ é compacta quanto para o caso em que $M$ é completa, não compacta, obtendo uma generalização das subvariedades rotacionais em codimensão 2 em formas espaciais. Em ambos os casos, faremos forte uso de uma caracterização local, obtida por Dajczer-Tojeiro (DAJCZER; TOJEIRO, 2004), de imersões isométricas $f: M^{n}=L^{p} \times_{\rho} N^{n-p} \rightarrow \mathbb{Q}_{c}^{n+2}$, com $n \geq 3$, de um produto warped de variedades Riemannianas.

Finalizamos esta introdução com uma breve descrição do conteúdo de cada capítulo. O Capítulo 2 foi incluído para tornar a leitura tão auto-suficiente quanto possível. Embora o conteúdo desse capítulo seja um assunto clássico na literatura, julgamos importante recapitular alguns resultados que serão usados frequentemente no decorrer do texto. Por se tratar de um capítulo de pré-requisitos, não apresentamos as demonstrações dos resultados, mas tomamos o cuidado de indicar ao leitor referências onde estas podem ser encontradas. As Seções 2.1 e 2.2 contêm resultados de teoria de subvariedades e ações de grupos de Lie, onde destacamos a Proposição 8 a respeito das curvatuas das órbitas principais de uma ação isométrica na variedade. Na seção 2.3 discutimos a noção de produto warped em uma variedade Riemanniana. O resultado principal desta seção é o Teorema 20, que responde a questão de saber quando que uma dada variedade Riemanniana, sob a ação de um grupo de Lie, admite uma estrutura de produto warped. A Seção 2.4 aborda o conceito de produto warped de imersões isométricas, generalizando o de produto Riemanniano. Resultados básicos, mas notáveis, são os teoremas devido a Moore (MOORE, 1971, Main Lemma) e Nölker (NÖLKER, 1996, Theorem 7), que dão condições necessárias para que uma imersão isométrica no espaço Euclidiano seja um produto Riemanniano e produto warped, respectivamente, de imersões isométricas. Finalmente, na Seção 2.5 recapitulamos o resultado central de Dajczer-Tojeiro (DAJCZER; TOJEIRO, 2004), que será usado nesta tese.

No capítulo 3 apresentamos os resultados obtidos na nossa tese. A Seção 3.1 contém alguns resultados auxiliares que são usados no decorrer do texto. Inicialmente, dividimos as imersões isométricas da forma $f: L^{p} \times_{\rho} N^{n} \rightarrow \mathbb{Q}_{c}^{l}$ em três grupos, A, B e C, em termos do 
subespaço do fibrado normal gerado pelos vetores horizontais e verticais. O resultado central dessa seção diz que se uma imersão isométrica $f: I \times{ }_{\rho} N^{n-1} \rightarrow \mathbb{Q}_{c}^{n+2}$ é do tipo B, então $I \times{ }_{\rho} N^{n-1}$ tem curvatura seccional constante igual a $c$ (cf. Proposição 16). Na Seção 3.2 apresentamos um resultado de natureza local, onde provaremos que, localmente, a imersão isométrica tem segunda forma fundamental adaptada a rede produto:

Proposição 1. Sejam $M^{n}$ uma variedade Riemanniana completa, com $n \geq 4$, e $G \subset \operatorname{Iso}\left(M^{n}\right)$ um subgrupo fechado agindo sobre $M^{n}$ com cohomogeneidade 1, de modo que as órbitas principais são hipersuperfícies umbílicas de $M$. Dado um ponto regular $p \in M^{n}$, seja $\Gamma \subset M^{n}$ o subconjunto aberto isométrico ao produto warped $I \times{ }_{\rho} N^{n-1}$, onde $I \subset \mathbb{R}$ é um intervalo aberto e $N$ é isométrico a um aberto da órbita $\Sigma_{p}^{n-1}$. Se $f: I \times{ }_{\rho} N^{n-1} \rightarrow \mathbb{Q}^{n+2}$ é uma imersão isométrica tal que $N^{n-1}$ não é flat, então a segunda forma fundamental de $f$ é adaptada a rede produto.

Nas seções seguintes apresentamos uma classificação global de tais subvariedades. $\mathrm{Na}$ Seção 3.3 consideramos o caso em que o ambiente é o espaço Euclidiano, e obtems o seguinte resultado no caso em que a variedade $M^{n}$ é compacta:

Teorema 1. Sejam $f: M^{n} \rightarrow \mathbb{R}^{n+2}$ uma imersão isométrica, com $M^{n}$ compacta, $n \geq 4$ e $G \subset$ Iso $\left(M^{n}\right)$ um subgrupo conexo, fechado agindo sobre $M^{n}$ com cohomogeneidade 1, de modo que as órbitas principais são hipersuperfícies umbílicas. Então $f\left(M^{n}\right)$ satifaz uma das seguintes possibilidades:

1. $f\left(M^{n}\right)$ é uma subvariedade rotacional;

2. $f\left(M^{n}\right)$ é um produto warped de hipersuperfícies.

No caso em que a variedade $M^{n}$ é completa não compacta, teremos dois subcasos a considerar. Inicialmente, supomos que o subgrupo $G$ é compacto e, em seguida, supomos que $G$ é fechado não compacto.

Teorema 2. Sejam $f: M^{n} \rightarrow \mathbb{R}^{n+2}$ uma imersão isométrica, com $M^{n}$ compacta, $n \geq 4$ e $G \subset$ Iso $\left(M^{n}\right)$ um subgrupo conexo, fechado agindo sobre $M^{n}$ com cohomogeneidade 1 , de modo que as órbitas principais são hipersuperfícies umbílicas.

1. Se $G$ é compacto, então $f\left(M^{n}\right)$ satifaz uma das seguintes possibilidades:

a) $f\left(M^{n}\right)$ é uma subvariedade rotacional;

b) $f\left(M^{n}\right)$ é um produto warped de hipersuperfícies;

c) $f\left(M^{n}\right)$ é um $(n-2)$-cilindro.

2. Se $G$ não é compacto, então $f\left(M^{n}\right)$ satisfaz uma das seguintes possibilidades:

a) $f\left(M^{n}\right)$ é um produto warped de hipersuperfíces, 
b) $f\left(M^{n}\right)$ é um $(n-2)$-cilindro.

Na Seção 3.4 consideramos o caso em que o ambiente é a esfera.

Teorema 3. Sejam $f: M^{n} \rightarrow \mathbb{S}^{n+2}$ uma imersão isométrica, com $M^{n}$ compacta, $n \geq 4$ e um subgrupo conexo e fechado $G \subset I s o\left(M^{n}\right)$ agindo sobre $M^{n}$ de modo que as órbitas principais de $G$ são hipersuperfícies de $M^{n}$ com cohomogeneidade 1 e umbílicas, então $f\left(M^{n}\right)$ satifaz uma das seguintes afirmativas:

1. $f\left(M^{n}\right)$ é uma subvariedade rotacional;

2. $f\left(M^{n}\right)$ é um produto warped de hipersuperfícies;

3. $f\left(M^{n}\right)$ é uma esfera.

No caso em que a variedade $M^{n}$ é completa não compacta, teremos dois subcasos a considerar, inicialmente vamos supor que o subgrupo $G$ é compacto, em seguida vamos supor que o subgrupo $G$ é fechado e não compacto, obtendo o seguinte resultado:

Teorema 4. Sejam $f: M^{n} \rightarrow \mathbb{S}^{n+2}$ uma imersão isométrica, com $M^{n}$ completa e não compacta, $n \geq 4$ e um subgrupo conexo, fechado $G \subset I s o\left(M^{n}\right)$ agindo sobre $M^{n}$ de modo que as órbitas principais de $G$ são hipersuperfícies de $M^{n}$ com cohomogeneidade 1 e umbílicas. Temos

1. Se $G$ é compacto, então $f\left(M^{n}\right)$ satifaz uma das seguintes afirmativas:

a) $f\left(M^{n}\right)$ é uma subvariedade rotacional;

b) $f\left(M^{n}\right)$ é um produto warped de hipersuperfícies;

c) $f\left(M^{n}\right)$ é uma esfera.

2. Se $G$ não é compacto, então $f\left(M^{n}\right)$ satisfaz uma das seguintes afirmativas:

a) $f\left(M^{n}\right)$ é produto warped de hipersuperfícies,

b) $f\left(M^{n}\right)$ é uma esfera.

Finalmente, na Seção 3.5 consideramos o caso em que o ambiente é o espaço hiperbólico.

Teorema 5. Sejam $f: M^{n} \rightarrow \mathbb{H}^{n+2}$ uma imersão isométrica, com $M^{n}$ compacta, $n \geq 4$ e um subgrupo conexo e fechado $G \subset I s o\left(M^{n}\right)$ agindo sobre $M^{n}$ de modo que as órbitas principais de $G$ são hipersuperfícies de $M^{n}$ com cohomogeneidade 1 e umbílicas, então $f\left(M^{n}\right)$ satifaz uma das seguintes afirmativas:

1. $f\left(M^{n}\right)$ é uma subvariedade rotacional elíptica;

2. $f\left(M^{n}\right)$ é um produto warped de hipersuperfícies: ou elíptico, ou hiperbólico, ou parabólico; 
3. $f(M)$ é um cone: hiperbólico ou parabólico.

No caso em que a variedade $M^{n}$ é completa não compacta, teremos dois subcasos a considerar. Inicialmente, supomos que o subgrupo $G$ seja compacto e, em seguida, o caso em que $G$ seja fechado não compacto.

Teorema 6. Sejam $f: M^{n} \rightarrow \mathbb{H}^{n+2}$ uma imersão isométrica, com $M^{n}$ completa e não compacta, $n \geq 4$ e um subgrupo conexo, fechado $G \subset I s o\left(M^{n}\right)$ agindo sobre $M^{n}$ de modo que as órbitas principais de $G$ são hipersuperfícies de $M^{n}$ com cohomogeneidade 1 e umbílicas. Temos

1. Se $G$ é compacto, então $f\left(M^{n}\right)$ satifaz uma das seguintes afirmativas:

a) $f\left(M^{n}\right)$ é uma subvariedade rotacional elíptica;

b) $f\left(M^{n}\right)$ é um cone: parabólico, ou hiperbólico;

c) $f\left(M^{n}\right)$ é um produto warped de hipersuperfícies: elíptica, ou parabólica, ou hiperbólica.

2. Se $G$ não é compacto, então $f\left(M^{n}\right)$ satisfaz uma das seguintes afirmativas:

a) $f(M)$ é uma subvariedade rotacional: hiperbólica ou parabólica.

b) $f\left(M^{n}\right)$ é um produto warped de hipersuperfícies: elíptica, ou parabólica, ou hiperbólica;

c) $f\left(M^{n}\right)$ é um cone: parabólico, ou hiperbólico. 



\section{SUBVARIEDADES E IMERSÕES ISOMÉTRICAS}

\subsection{Subvariedades}

Nesta seção recapitularemos alguns resultados fundamentais da teoria de subvariedades que podem ser encontrados no excelente livro (DAJCZER et al., 1990).

Uma imersão $f: M^{n} \rightarrow \tilde{M}^{n+p}$ entre duas variedades Riemannianas é dita ser uma imersão isométrica se

$$
\left\langle f_{*} X, f_{*} Y\right\rangle=\langle X, Y\rangle,
$$

para quaisquer $X, Y \in T M$. A segunda forma fundamental de $f$, denotada por $\alpha_{f}$, é a aplicação bilinear simétrica $\alpha_{f}: T M \times T M \rightarrow T M^{\perp}$ definida por

$$
\alpha_{f}(X, Y)=\left(\tilde{\nabla}_{X} Y\right)^{\perp}
$$

para quaisquer $X, Y \in T M$, onde $\tilde{\nabla}$ denota a conexão de Levi-Civita de $\tilde{M}$. Temos então a primeira fórmula da teoria de subvariedades, conhecida como a fórmula de Gauss

$$
\tilde{\nabla}_{X} f_{*} Y=f_{*} \nabla_{X} Y+\alpha_{f}(X, Y),
$$

onde $\nabla$ denota a conexão de Levi-Civita de $M$.

O operador de forma da imersão $f$ no ponto $x \in M$, relativo a um vetor normal $\xi \in$ $T_{x} M^{\perp}$, é o operador linear auto-adjunto $A_{\xi}: T_{x} M \rightarrow T_{x} M$ dado por

$$
\left\langle A_{\xi} X, Y\right\rangle=\left\langle\alpha_{f}(X, Y), \xi\right\rangle,
$$

para quaisquer $X, Y \in T_{x} M$. O operador $A$ permite escrever a segunda fórmula básica, conhecida como a fórmula de Weingarten, e dada por

$$
\tilde{\nabla}_{X} \xi=-f_{*} A_{\xi} X+\nabla_{X}^{\perp} \xi
$$


para quaisquer $X \in T M$ e $\xi \in T M^{\perp}$.

Usando as fórmulas de Gauss e Weingartein, podemos obter as equações de compatibilidade de uma imersão isométrica. Mais precisamente, se $R$ e $\tilde{R}$ denotam os tensores de curvatura de $M^{n}$ e $\tilde{M}^{n+p}$, respectivamente, as equações de Gauss, Codazzi e Ricci da imersão isométrica $f: M^{n} \rightarrow \tilde{M}^{n+p}$ são dadas, respectivamente, por

$$
\begin{aligned}
\langle R(X, Y) Z, W\rangle= & \langle\tilde{R}(X, Y) Z, W\rangle+\left\langle\alpha_{f}(X, W), \alpha_{f}(Y, Z)\right\rangle-\left\langle\alpha_{f}(X, Z), \alpha_{f}(Y, W)\right\rangle, \\
& (\tilde{R}(X, Y) Z)^{\perp}=\left(\nabla_{X}^{\perp} \alpha_{f}\right)(Y, Z)-\left(\nabla_{Y}^{\perp} \alpha_{f}\right)(X, Z)
\end{aligned}
$$

$\mathrm{e}$

$$
\left\langle R^{\perp}(X, Y) \xi, \eta\right\rangle=\left\langle\tilde{R}^{\perp}(X, Y) \xi, \eta\right\rangle+\left\langle\left[A_{\xi}, A_{\eta}\right] X, Y\right\rangle,
$$

para quaisquer $X, Y, Z, W \in T M$ e $\xi, \eta \in T M^{\perp}$.

Quando $M^{n+p}$ é uma variedade Riemanniana com curvatura seccional constante igual a $c$, as equações de Gauss, Codazzi e Ricci tornam-se

$$
\begin{gathered}
\langle R(X, Y) Z, W\rangle=c\langle(X \wedge Y) Z, W\rangle+\left\langle\alpha_{f}(X, W), \alpha_{f}(Y, Z)\right\rangle-\left\langle\alpha_{f}(X, Z), \alpha_{f}(Y, W)\right\rangle, \\
\left(\nabla_{X}^{\perp} \alpha_{f}\right)(Y, Z)=\left(\nabla_{Y}^{\perp} \alpha_{f}\right)(X, Z)
\end{gathered}
$$

e

$$
\left\langle R^{\perp}(X, Y) \xi, \eta\right\rangle=\left\langle\left[A_{\xi}, A_{\eta}\right] X, Y\right\rangle,
$$

respectivamente, onde $X \wedge Y$ denota o produto wedge dado por

$$
(X \wedge Y) Z=\langle Y, Z\rangle X-\langle X, Z\rangle Y \text {. }
$$

O subespaço de nulidade relativa de uma imersão isométrica $f: M^{n} \rightarrow \tilde{M}^{n+p}$ num ponto $x \in M$, denotado por $\Delta(x)$, é o núcleo da segunda forma fundamental no ponto $x$ :

$$
\Delta(x)=\left\{X \in T_{x} M: \alpha_{f}(X, Y)=0, \forall Y \in T_{x} M\right\} .
$$

De forma equivalente,

$$
\Delta(x)=\bigcap_{\xi \in T_{x} M^{\perp}} \operatorname{kerA} \xi .
$$

A dimensão de $\Delta(x)$, denotada por $v(x)$, é chamada o índice de nulidade relativa de $f$ em $x$. Denotamos ainda por $v_{0}$ o índice de nulidade relativa mínima de $f$. O ponto importante aqui é que, para qualquer imersão isométrica $f: M^{n} \rightarrow \tilde{M}^{n+p}$, o índice de nulidade relativa $v$ é uma função semi-contínua superiormente. Em particular, o subconjunto

$$
M_{0}=\left\{x \in M: v(x)=v_{0}\right\},
$$

onde $v$ atinge seu valor mínimo $v_{0}$, é aberto em $M$. Além disso, a distribuição de nulidade relativa $x \mapsto \Delta(x)$ é diferenciável em qualquer subconjunto aberto de $M^{n}$ onde $v$ é constante (DAJCZER et al., 1990, Proposition 1.18). 
A proposição seguinte nos dá condições necessárias para a existência de hipersuperfícies de curvatura seccional constante em formas espaciais em termos da nulidade.

Proposição 2 ((GONÇALVES, 2006, Proposição 10)). Para qualquer hipersuperfície $f: M_{\tilde{c}}^{n} \rightarrow$ $\tilde{M}_{c}^{n+1}, \operatorname{com} n \geq 3$, uma das seguintes possibilidades ocorre:

(i) $\tilde{c}=c$ e $v \geq n-1$;

(ii) $\tilde{c}>c$ e $f$ é umbílica (não totalmente geodésica).

Uma imersão isométrica $f: M^{n} \rightarrow \mathbb{Q}_{c}^{n+p}$ é dita ter fibrado normal flat quando $R^{\perp} \equiv 0$, onde $R^{\perp}$ denota o tensor de curvatura do fibrado normal $T M^{\perp}$ de $f$. Pela equação de Ricci, $f$ tem fibrado normal flat se, e somente se, os operadores de forma

$$
\left\{A_{\xi}: \xi \in T M^{\perp}\right\}
$$

são simultaneamente diagonalizáveis ou, equivalentemente, se existe uma base ortonormal $\left\{X_{1}, \ldots, X_{n}\right\}$ de $T_{x} M$ tal que

$$
\alpha_{f}\left(X_{i}, X_{j}\right)=0
$$

para quaisquer $1 \leq i \neq j \leq n$.

Proposição 3 ((DAJCZER et al., 1990, Proposition 1.24)). Uma imersão isométrica $f: M^{n} \rightarrow$ $\tilde{M}_{c}^{m}$ tem fibrado normal flat em um ponto $x \in M^{n}$ se, e somente se, os operadores forma $\left\{A_{\xi}\right.$ : $\left.\xi \in T_{x} M^{\perp}\right\}$ são simultaneamente diagonalizáveis ou, equivalentemente, se, e somente se, existe uma base ortonormal $\left\{X_{1}, \ldots, X_{n}\right\}$ de $T_{x} M$ tal que

$$
\alpha\left(X_{i}, X_{j}\right)=0
$$

para $i \neq j$.

Portanto, em cada ponto $x \in M^{n}$ onde $R^{\perp}(x)=0$, o espaço tangente $T_{x} M$ decompõe-se ortogonalmente como

$$
T_{x} M=E_{1}(x) \oplus E_{2}(x) \oplus, \ldots, \oplus E_{S}(x),
$$

com a propriedade de que, para cada $\xi \in T_{x} M^{\perp}$, existe um número real $\lambda_{i}(\xi)$, com $1 \leq i \leq s=$ $s(x)$, tal que

$$
\left.A_{\xi}\right|_{E_{i}(x)}=\lambda_{i}(\xi) I
$$

e as aplicações $\xi \mapsto \lambda_{i}(\xi)$ são duas a duas distintas. Como tais aplicações são lineares, existem únicos e distintos vetores $\eta_{i}(x) \in T_{x} M^{\perp}$, com $1 \leq i \leq s$, chamados as normais principais de $f$ em $x$ tais que

$$
\lambda_{i}(x)=\left\langle\eta_{i}(x), \xi\right\rangle
$$

Portanto,

$$
E_{i}(x)=E_{\eta_{i}(x)}=\left\{X \in T_{x} M: \alpha_{f}(X, Y)=\langle X, Y\rangle \eta_{i}(x), \forall Y \in T_{x} M\right\}
$$


e a segunda forma fundamental de $f$ satisfaz

$$
\alpha_{f}(X, Y)=\sum_{i=1}^{s} \alpha_{f}\left(X_{i}, Y_{j}\right) \eta_{i}(x)
$$

onde $X \mapsto X_{i}$ é a projeção ortogonal de $X$ sobre $E_{i}(x)$.

Pela equação de Gauss, a curvatura seccional $K$ de $M^{n}$ satisfaz

$$
K(X, Y)=c+\left\langle\eta_{i}(x), \eta_{j}(x)\right\rangle
$$

ao longo do plano gerado por $X \in E_{i}(x)$ e $Y \in E_{j}(x)$, com $1 \leq i \neq j \leq s$, enquanto que

$$
K(X, Y)=c+\left\|\eta_{i}(x)\right\|^{2},
$$

ao longo do plano gerado por $X, Y \in E_{i}(x)$.

Observação 1. Quando a imersão isométrica $f$ tem codimensão 2 e $f$ não é totalmente geodésica, tem-se

$$
T_{x} M=E_{1}(x) \oplus E_{2}^{n-1}(x),
$$

para todo $x \in M$. De fato, pela decomposição acima, obtemos $\eta_{1}(x) \neq \eta_{2}(x)$. Se $\operatorname{dim} E_{1}(x) \geq 2$ e $\operatorname{dim} E_{2}(x) \geq 2$, então por (2.3) segue que $\eta_{1}(x)=\eta_{2}(x)=0$, que é uma contradição. Assim, ou $\operatorname{dim} E_{1}(x)=1$ ou $\operatorname{dim} E_{2}(x)=1$, e isso prova a decomposição (2.4).

Dado um espaço vetorial $V$, considere formas bilineares e simétricas $\phi_{1}, \ldots, \phi_{n}$ em $V$. Dizemos que $\phi_{1}, \ldots \phi_{n}$ são exteriormente ortogonais, se

$$
\sum_{i=1}^{n}\left[\phi_{i}(X, Y) \phi_{i}(Z, W)-\phi_{i}(X, W) \phi_{i}(Y, Z)\right]=0
$$

para quaisquer $X, Y, Z, W \in V$.

Teorema 7 ((MOORE, 1972, Theorem 1)). Sejam $\phi_{1}, \ldots, \phi_{n}$ formas bilineares, simétricas e exteriormente ortogonais em um espaço vetorial $V$, com a seguinte propriedade: se $X$ é um vetor em $V$ tal que $\phi_{i}(X, Y)=0$, para todo $1 \leq i \leq n$ e todo $Y \in V$, então $X=0$. Então existe uma matriz real ortogonal $\left(a_{i, j}\right)$ e $n$ funcionais lineares $\xi_{1}, \ldots, \xi_{n}$ tais que

$$
\phi_{i}=\sum_{j=1}^{n} a_{1, j} \xi_{i} \bigotimes \xi_{j}
$$

com $1 \leq i \leq n$. Disso decorre que $\phi_{1}, \ldots, \phi_{n}$ são simultaneamente diagonalizáveis em relação à base dual de $\xi_{1}, \ldots, \xi_{n}$.

O teorema seguinte dá condições, em termos das curvaturas da subvariedade e do ambiente, a fim de garantir a existência de imersão isométrica de uma variedade Riemanniana compacta em uma variedade Riemanniana simplesmente conexa. 
Teorema 8 ((O’NEILL, 1960, Theorem 1)). Seja $\tilde{M}$ uma variedade Riemanniana completa e simplesmente conexa, com $K_{\tilde{M}} \leq 0$. Se $M$ é uma variedade Riemanniana compacta, com $\operatorname{dim} \tilde{M}<2 \cdot \operatorname{dim} M$ e $K_{M} \leq K_{\tilde{M}}$, então não existe imersão isométrica $f: M \rightarrow \tilde{M}$.

O próximo resultado é um teorema devido a Philip Hartman para imersões isométricas de variedades com curvatura não-negativa no espaço Euclidiano.

Teorema 9 ((HARTMAN, 1965, Corollary 7.16)). Se $f: M^{n} \rightarrow \mathbb{R}^{n+p}$ é uma imersão isométrica de uma variedade Riemanniana completa e flat, $\operatorname{com} n \geq 2$ e $1 \leq p \leq n-1$, então $f$ é um $(n-p)$-cilindro.

O seguinte resultado nos dá condições para a existência de uma imersão isométrica de uma variedade Riemanniana compacta sobre uma variedade Riemanniana simplesmente conexa, onde as condições das curvaturas seccionais são modificadas.

Teorema 10 ((MOORE, 1972, Theorem 3.1)). Seja $\tilde{M}$ uma variedade Riemanniana completa, simplesmente conexa, cujas curvaturas seccionais satisfazem

$$
K_{\tilde{M}} \leq b \leq 0
$$

Seja $M$ uma variedade Riemanniana compacta cujas curvaturas seccionais satisfazem $K_{M}-K_{\tilde{M}} \leq$ $-b$. Se $\operatorname{dim} \tilde{M}<2 \cdot \operatorname{dim} M$, então não existe imersão isométrica $f: M \rightarrow \tilde{M}$.

O teorema seguinte nos dá informações sobre as curvaturas da subvariedade e do ambiente em termos da codimensão da imersão isométrica.

Teorema 11 ((DAJCZER et al., 1990, Theorem 5.1)). Seja $f: M_{c}^{n} \rightarrow \mathbb{Q}_{\tilde{c}}^{n+p}$ uma imersão isométrica. Então os seguintes fatos são verdadeiros:

(i) Se $c<\tilde{c}$ então $p \geq n-1$;

(ii) Se $c>\tilde{c}$ e $p \leq n-2$, então para cada $x \in M^{n}$ existem um vetor unitário $\xi \in T_{x} M^{\perp}$ e uma forma bilinear flat

$$
\gamma(x): T_{x} M \times T_{x} M \rightarrow\{\xi\}^{\perp} \subset T_{x} M
$$

tal que

$$
\alpha(x)=\gamma(x)+\sqrt{c-\tilde{c}}\langle,\rangle \xi
$$

O teorema a seguir, devido a Joseph Erbacher, nos dá informações sobre o tensor de curvatura e o índice de nulidade de uma imersão isométrica de codimensão 2 entre variedades Riemannianas de curvatura seccional constante.

Teorema 12 ((ERBACHER, 1972, Theorem 2)). Seja $f: M_{c}^{n} \rightarrow N_{\tilde{c}}^{n+2}$ uma imersão isométrica entre variedades Riemannianas de curvatura seccional constante, com $n \geq 3$. As seguintes afirmações são verdadeiras: 
(i) Se $c \neq \tilde{c}$, então $f$ tem fibrado normal flat.

(ii) Se $c=\tilde{c}$, então para cada $x \in M^{n}$, ou $f$ tem fibrado normal flat em cada $x$, ou o índice de nulidade relativa de $f$ em $x$ é $n-2$.

Teorema 13 ((STIEL, 1965, Theorem 2)). Sejam $M^{n}$ e $N^{n+p}$ variedades Riemannianas, com mesma curvatura seccional constante $c$. Suponhamos que $M^{n}$ seja completa. Se $f: M^{n} \rightarrow N^{n+p}$ é uma imersão isométrica, com $n \geq 2 p$, então $f$ é totalmente geodésica.

Seja $M$ uma variedade Riemanniana. Um subfibrado vetorial $E \subset T M$ é dito ser paralelo se $\nabla_{X} Y \in E$, para quaisquer $X \in T M$ e $Y \in \Gamma(E)$. Dizemos que $E$ é totalmente geodésico se $\nabla_{X} Y \in \Gamma(E)$, para quaisquer $X, Y \in \Gamma(E)$. Mais geralmente, se existe $\eta \in \Gamma\left(E^{\perp}\right)$ tal que $\left(\nabla_{X} Y\right)_{E^{\perp}}=\langle X, Y\rangle \eta$, quaisquer que sejam $X, Y \in \Gamma(E)$, dizemos que $E$ é totalmente umbílico. Se além disso, $\left(\nabla_{X} \eta\right)_{E^{\perp}}=0$, para todo $X \in E, E$ é dito um subfibrado esférico.

Se $E$ é um subfibrado vetorial totalmente umbílico (totalmente geodésico, ou paralelo) então $E$ é automaticamente integrável. Além disso, as folhas $\sigma$ de $E$ são subvariedades umbílicas (totalmente geodésicas se $E$ é paralelo ou totalmente geodésico) de $M^{n}$ com campo curvatura média $\left.\eta\right|_{\sigma}$. O subfibrado $E$ ser esférico significa que cada folha $\sigma$ de $E$ é uma subvariedade esférica de $M^{n}$, i.e., $\sigma$ é uma subvariedade umbílica de $M^{n}$ cujo campo curvatura média $\left.\eta\right|_{\sigma}$ é paralelo com relação à conexão normal

$$
\nabla_{X}^{\perp} \eta=\left(\nabla_{X} \eta\right)_{E^{\perp}}=0
$$

para todo $X \in E$.

\subsection{Ações de grupos de Lie}

Nesta seção recapitularemos alguns resultados sobre ações de grupos de Lie que serão usados ao longo deste trabalho, que podem ser encontrados em (BERNDT; CONSOLE; OLMOS, 2016), (PALAIS; TERNG, 2006), (PALAIS; TERNG, 1987).

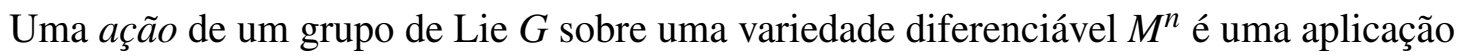
diferenciável $A: G \times M \rightarrow M$ satisfazendo

$$
A(g, A(h, p))=A(g h, p) \quad \text { e } \quad A(e, p)=p,
$$

para todo $p \in M$ e quaisquer $g, h \in G$, onde $e$ é a identidade de $G$. Dizemos também que $G$ age sobre $M^{n}$ ou que $M^{n}$ é uma $G$-variedade. Note que $A$ induz um homomorfismo de grupos $\phi: G \rightarrow \operatorname{Diff}(M)$ dado por

$$
g \in G \mapsto \phi(g) \in \operatorname{Diff}(\mathrm{M}),
$$

onde $\phi(g): M^{n} \rightarrow M^{n}$ é definido por $\phi(g)(x)=A(g, x)$, para todo $x \in M^{n}$. Assim, uma ação de um grupo de Lie $G$ sobre uma variedade $M^{n}$ pode ser vista como uma representação $\phi: G \rightarrow \operatorname{Diff}(M)$ de $G$ como um subgrupo de $\operatorname{Diff}(M)$, e escreve-se $g(x)$ em vez de $A(g, x)$. 
Dado uma ação $A: G \times M \rightarrow M$, o subgrupo de isotropia de $G$ em $p \in M$ é o subgrupo $G_{p}$ de $G$ definido por

$$
G_{p}=\{g \in G ; g(p)=p\}
$$

$G_{p}$ age de modo natural sobre o espaço tangente a $M$ em $p$ através da representação

$$
g \in G_{p} \mapsto d g(p) \in G L\left(T_{p} M\right)
$$

chamada representação de isotropia de $G$ em $p$.

Teorema 14 (Myers-Steenrod). O grupo $I s o(M)$ tem a estrutura de grupo de Lie em relação à topologia compacto-aberta, de modo que a isotropia, em qualquer ponto, é compacto. Se $M$ é compacta o mesmo vale para $I s o(M)$. Além disso, a ação natural de $I s o(M)$ em $M$ é diferenciável.

Se M é uma variedade Riemanniana, todo homomorfismo de grupos de Lie $\phi: G \rightarrow$ Iso $(M)$ determina uma ação sobre $M$, e neste caso dizemos que $M$ é uma $G$-variedade Riemanniana. Dado uma ação $A: G \times M \rightarrow M$ e fixado um ponto $p \in M$, a órbita de $p$ em $M$, determinada pela ação de $G$ em $M$, é o subconjunto $G(p)$ de $M$ dado por

$$
G(p)=\left\{g(p) \in M^{n} ; g \in G\right\} .
$$

Se $G(p)=M$, para algum $p \in M$, dizemos que $M$ é $G$-homogênea.

Definição 1. Dado um ponto $x \in M$, uma subvariedade $S$ de $M$ é chamada um slice no ponto $x$ se existem uma vizinhança $G$-invariante $U$ de $x$ e uma retratação $G$-equivariante $r: S \rightarrow G(x)$ tal que $S=r^{-1}(x)$. O aberto $\Gamma=G(S)$ é chamado o tubo $G$-invariante em torno da órbita $G(x)$.

Teorema 15. Dados uma variedade Riemanniana $M$ e um subgrupo fechado $G \subset I s o(M)$, a ação natural $A: G \times M \rightarrow M$ satisfaz as seguintes propriedades:

(i) $G(p)$ é uma subvariedade fechada e mergulhada de $M$, para todo $p \in M$,

(ii) Para todo $p \in M^{n}$, existe $r \geq 0$ tal que

$$
U=\left\{\exp _{x}(v): x \in G(p) \text { e } v \in T_{x} G(p)^{\perp},\|v\|<r\right\}
$$

é uma vizinhaça tubular $G$-invariante de $G(p)$.

Sejam $M$ uma variedade Riemanniana e $G \subset I s o(M)$. Dado uma componente conexa $M^{i}$ de $M$, e vendo os subgrupos de isotropia também como subvariedades, seja $m=\min \left\{\operatorname{dim} G_{p}\right.$ : $\left.p \in M^{i}\right\}$ e $k$ o menor número de componentes conexas de um subgrupo de isotropia dentre todos os subgrupos de isotropia de dimensão $m$. Dado $p \in M^{i}$, dizemos que $G(p)$ é uma órbita principal de $M$ se a subvariedade $G_{p}$ tem dimensão $m$ e o número de componentes conexas de $G_{p}$ é $k$. Em particular, uma órbita principal de $M^{i}$ tem dimensão máxima dentre todas as órbitas de $M^{i}$. 
Se $p \in M^{i}$ e $G(p)$ tem dimensão maximal dentre todas as órbitas de $M^{i}$, mas não é principal, então $G(p)$ é chamada órbita excepcional. Se $G(p)$ não tem dimensão maximal, é chamada órbita singular. A codimensão de uma órbita principal como subvariedade de $M^{n}$ será chamada cohomogeneidade de $M^{n}$. Se $G(p)$ é uma órbita principal, o ponto $p$ chama-se ponto regular de $M^{n}$. Denotaremos o conjunto dos pontos regulares por $M_{\text {reg }}$.

Teorema 16. Sejam $M$ uma variedade Riemanniana e $G \subset I s o(M)$ um subgrupo fechado e conexo. Então, a parte regular da variedade $M$ é um subconjunto aberto, conexo e denso em $M$. Além disso, dado um ponto $p \in M$, as seguintes afirmações são equivalentes:

(i) $p \in M_{\text {reg }}$,

(ii) Existe uma vizinhança $V$ de $p$ em $M$ tal que para todo $x \in V$, existe $g \in G$ tal que $G_{p} \subset G_{A(g, x)}=g G_{x} g^{-1}$.

(iii) $g_{*} \psi=\psi$, para todo $g \in G_{p}$ e para todo $\psi \in T_{p} G(p)^{\perp}$.

Corolário 1. Dados $p \in M_{\text {reg }}$ e $x \in \exp _{p}\left(T_{p} G(p)^{\perp}\right)$, então $G_{p} \subset G_{x}$. Em particular, tal $x$ é regular se, e somente se, $G_{x}=G_{p}$.

Teorema 17. Sejam $M$ uma variedade Riemanniana e $G \subset I s o(M)$ um subgrupo tal que as órbitas de $G$ são hipersuperfícies umbílicas de $M$ com cohomogeneidade 1 . Se $G$ é compacto, então o espaço das órbitas $\Omega=M / G$ é homeomorfo a um dos seguintes espaços:

(i) O circulo $\mathbb{S}^{1}$

(ii) O intervalo aberto $(0,1)$,

(iii) O intervalo semi-aberto $[0,1)$,

(iv) O intervalo fechado $[0,1]$.

Como consequência do Teorema 17 concluimos que nos itens (i) e (ii) existem somente órbitas principais; no item (iii) existe apenas uma órbita que não é principal, e no item (iv) existem duas órbitas que não são principais.

Recordaremos agora a definição de geodésica normal de uma $G$-variedade de cohomogeneidade 1. Sejam $M$ uma variedade Riemanniana completa e $G \subset I s o(M)$ um subgrupo fechado. Uma subvariedade imersa completa $S \subset M$ é dita uma seção de $M$ se $S$ intercepta todas as órbitas de $M$ e sempre ortogonalmente. Denotaremos $S \cap M_{\text {reg }}$ por $S_{r}$.

Definição 2. Sejam $M$ uma $G$-variedade de cohomogeneidade 1. Uma geodésica $\gamma:(a, b) \rightarrow M$, parametrizada pelo comprimento de arco, é chamada uma geodésica normal se o vetor $\gamma^{\prime}(t)$ é ortogonal à órbita $\Sigma_{\gamma(t)}^{n-1}$ no ponto $\gamma(t)$, para todo $t \in(a, b)$. 
As geodésicas normais apresentam várias propriedades importantes. O teorema a seguir explicita algumas delas.

Teorema 18. Sejam $M$ uma variedade Riemanniana e $G \subset I s o(M)$ um subgrupo tal que as órbitas de $G$ são hipersuperfícies de cohomogeneidade 1 sobre $M$. Dado uma geodésica normal $\lambda:(-\delta, \delta) \rightarrow M$, valem as seguintes propriedades:

(i) $\lambda_{g}=g \circ \lambda$ é uma geodésica normal, para todo $g \in G$;

(ii) Se $p=\lambda(0) \in M_{\text {reg }}$, existe $0<\varepsilon \leq \delta$ tal que $\lambda(-\varepsilon, \varepsilon)$ é um slice em $p$;

(iii) $\operatorname{Se} S=\lambda(-\varepsilon, \varepsilon)$ é um slice no ponto regular $p=\lambda(0)$, então:

a) os subgrupos de isotropia $G_{\lambda(t)}$ são constantes, i.e., $G_{\lambda(t)}=G_{p}$, sempre que $-\varepsilon<$ $t<\varepsilon$

b) a aplicação $\Phi:(-\varepsilon, \varepsilon) \times G(p) \rightarrow \Gamma$ dada por $\Phi(t, g(p))=g(\lambda(t))$ é um difeomorfismo;

c) está bem definido o campo vetorial $\eta \in \mathfrak{X}(\Gamma)$ dado por

$$
\eta(y)=d g_{\lambda(t)}\left(\lambda^{\prime}(t)\right)
$$

com $y=g(\lambda(t))$ e $-\varepsilon<t<\varepsilon$. Além disso, $\eta$ é unitário e sua restrição a cada órbita principal contida em $\Gamma$ é ortogonal a esta órbita;

(iv) Se $M$ é completa, então a extensão de $\lambda$ a $\mathbb{R}$ é uma geodésica normal cuja imagem intercepta toda órbita de $G$ e, portanto, $M=G(\mathbb{R})$.

Sejam $M$ uma variedade Riemanniana e $G \subset I s o(M)$ um subgrupo fechado. Dizemos que a ação natural de $G$ em $M$ é localmente polar, se a distribuição

$$
p \in M_{\text {reg }} \mapsto T_{p} G(p)^{\perp}
$$

é integrável. Diz-se que $M$ é uma $G$-variedade localmente polar.

Exemplo 1. Toda ação de cohomogeneidade 1 é localmente polar. Em particular, a ação natural de $O(n)$ em $\mathbb{R}^{n}$ é localmente polar, pois as órbitas são esferas.

Teorema 19. (BERNDT; CONSOLE; OLMOS, 2016) Se $M$ é uma $G$-variedade localmente polar, então $M$ admite seções. Além disso, se $S$ é uma seção de $M$, então $S_{r}$ é uma variedade integral. Em particular, uma seção de $M$ é uma subvariedade totalmente geodésica de $M$.

Se as seções de uma ação localmente polar são subvariedades mergulhadas e fechadas, dizemos que a ação é polar.

Exemplo 2. Vejamos alguns exemplos: 
(i) Toda ação localmente polar em $\mathbb{Q}^{n}$ é polar, pois toda subvariedade totalmente geodésica de $\mathbb{Q}^{n}$ é mergulhada e fechada.

(ii) Se $\mathbb{E} \subset \mathbb{R}^{n}$ é um subespaço vetorial e $G$ é o grupo das translações de vetores em $E$, então a ação é polar.

O resultado a seguir nos diz como determinar a parte regular de uma variedade sob a ação de um grupo $G \subset I s o(M)$.

Proposição 4. Sejam $M$ uma $G$-variedade localmente polar, completa, conexa e $S$ uma seção de $M^{n}$. Se $L$ é uma componente conexa de $S_{r}$, então $G(L)=M_{\text {reg }}$.

Fixado $p \in M^{n}$ e olhando $G(p)$ como subvariedade de $M^{n}$, um campo normal $\psi \in$ $T G(p)^{\perp}$ é chamado $G$-equivariante. Se $M$ é uma $G$-variedade localmente polar, então todo campo vetorial normal $G$-equivariante em uma órbita principal é $\nabla^{\perp}$-paralelo. Em particular, o campo vetor curvatura média da inclusão $i: G(p) \rightarrow M^{n}$ é $\nabla^{\perp}$-paralelo.

Proposição 5. (PALAIS; TERNG, 1987) Todo campo normal $G$-equivariante em uma órbita principal é $\nabla^{\perp}$-paralelo.

Dado uma órbita principal $G(p)$ e um vetor normal $\xi \in T_{p} G(p)^{\perp}$, está bem definido um campo normal $G$-equivarinate determinado por $\xi$ dado por

$$
\tilde{\xi}(g(p))=g_{*} \xi
$$

para todo $g \in G$. Se $A$ denota a segunda forma fundamental de $G(p)$ então, dados $g \in G, x \in G(p)$, $X, Y \in T_{x} G(p)$ e $\psi \in T_{x} G(p)^{\perp}$, temos:

$$
\begin{aligned}
\left\langle A_{\xi} X, Y\right\rangle & =\langle\alpha(X, Y), \xi\rangle=\left\langle\nabla_{X} Y-\nabla_{X} Y, \xi\right\rangle=\left\langle g_{*} \nabla_{X} Y, g_{*} \xi\right\rangle \\
& =\left\langle\nabla_{g_{*} X} g_{*} Y, \xi\right\rangle=\left\langle A_{g_{*}} g_{*} X, g_{*} Y\right\rangle \\
& =\left\langle g_{*}^{-1} A_{g_{*}} X, Y\right\rangle,
\end{aligned}
$$

o que implica

$$
A_{\tilde{\xi}(g(p))}=g_{*} A_{\xi} g_{*}^{-1},
$$

para todo $g \in G$. Como consequência obtemos o seguinte resultado.

Proposição 6. As curvaturas principais de uma órbita principal são constantes ao longo das direções normais $G$-equivariantes.

Proposição 7. (GONÇALVES, 2006, Proposição 50) Seja $M^{n}$ uma $G$-variedade localmente polar completa, onde $G$ é conexo. Se as órbitas principais de $G$ tem cohomogeneidade k, com $1 \leq k \leq n$ e são umbílicas sobre $M$, então elas são homotéticas entre si. 
Proposição 8. Seja $M$ uma $G$-variedade Riemanniana compacta de cohomogeneidade 1 e órbitas principais umbílicas. Então, vale somente uma das seguintes alternativas:

(a) Todas as órbitas principais têm curvatura seccional constante igual a $c$. Além disso, se $p \in M$ é um ponto regular, então as curvaturas seccionais em cada ponto $p \in G(p)$ são iguais a $c+k^{2}(p)$, onde $k(p)$ denota o autovalor do operador de Weingartein da inclusão $i: G(p) \rightarrow M$, que é constante ao longo de $G(p)$.

(b) Não existe órbita principal contendo pontos de curvatura seccional constante.

Demonstração. Suponhamos que o item (b) não ocorre, i.e., existem $p \in M_{\text {reg }}$ e $q \in G(p)$ tais que todas as curvaturas seccionais em $q$ são constantes. Pela homogeneidade de $G(p)$, temos que as curvaturas seccionais são constantes ao longo de $G(p)$. Como as órbitas principais são homotéticas entre si, obtemos que todas as órbitas principais têm curvatura seccional constante. Mostremos agora que, em cada ponto regular $p$, de curvatura seccional constante igual a $c$, as curvaturas seccionais da órbita $G(p)$ são iguais a $c+k^{2}(p)$. De fato, o autovalor $k(p)$ é constante ao longo da órbita $G(p)$. Assim, se $M$ tem curvatura seccional constante igual a $c$, a equação de Gauss, associada à inclusão umbílica $i: G(p) \rightarrow M$, implica que

$$
K_{G(p)}^{n-1}=c+k^{2}(p)
$$

e isso finaliza a demonstração.

\subsection{Produto warped de variedades Riemannianas}

Nesta seção discutiremos a noção de produto warped entre variedades Riemannianas, generalizando a noção de produto Riemanniano. Para os objetivos desta tese consideraremos variedades produto com apenas dois fatores, embora a maioria dos resultados possam ser estendidos para variedades produto com vários fatores.

Convencionaremos, inicialmente, a terminologia básica. Dado uma variedade produto $L \times N$, considere as projeções canônicas $\pi_{L}: L \times N \rightarrow L$ e $\pi_{N}: L \times N \rightarrow N$, bem como as subvariedades $L \times\{y\}$ e $\{x\} \times N$ de $L \times N$, onde $x$ e $y$ variam em $L$ e $N$, respectivamente. Estas subvariedades determinam folheações em $L \times N$, chamadas folheações canônicas induzidas por $L$ e $N$, respectivamente. O subfibrado vetorial de $T(L \times N)$ formado pelos espaços tangentes das subvariedades integrais da folheação canônica induzida por $L$ é chamado subfibrado horizontal e denotado por $\mathscr{H}$. De maneira análoga se define o subfibrado vertical $\mathscr{V}$. Deste modo, chamare$\operatorname{mos}\{x\} \times N=\pi_{L}^{-1}(x)$ de fibras e $L \times\{y\}=\pi_{N}^{-1}(y)$ de folhas. O levantamento horizontal de um vetor $\tilde{X} \in T_{x} L$ ao espaço tangente $T_{(x, y)} L \times N$ é o único vetor $X \in \mathscr{H}_{(x, y)}$ tal que $\pi_{L_{*}} X=\tilde{X}$. De maneira análoga se define o levantamento vertical de um vetor de $T N$.

Considere agora duas variedades Riemannianas $L$ e $N$ munidas de métricas $\langle,\rangle_{L}$ e $\langle,\rangle_{N}$, respectivamente. Uma métrica Riemanniana $\langle$,$\rangle na variedade produto L \times N$ é dita ser uma 
métrica produto warped das métricas $\langle,\rangle_{L}$ e $\langle,\rangle_{N}$ se existe uma função positiva $\rho: L \rightarrow \mathbb{R}$, chamada função warping, tal que

$$
\langle., .\rangle=\pi_{L}^{*}\langle., .\rangle_{L}+\left(\rho \circ \pi_{L}\right)^{2} \pi_{N}^{*}\langle., .\rangle_{N} .
$$

Neste caso, o par $(L \times N,\langle\rangle$,$) chama-se variedade produto warped e a denotamos simplesmente$ por $M=L \times{ }_{\rho} N$. Note que uma variedade produto Riemanniano é um caso particular da situação acima, no qual a função warping $\rho$ é constante e igual a 1. Se $\rho$ é uma função constante, não necessariamente igual a 1, a variedade produto warped é essencialmente um produto Riemanniano, basta corrigir a métrica do segundo fator.

Em uma variedade produto warped $L \times{ }_{\rho} N$, as folhas são todas isométricas à variedade $L$ pois, dado $y \in N$, a aplicação $x \in L \mapsto(x, y) \in L \times\{y\}$ é uma isometria, enquanto que as fibras são homotéticas à variedade $N$, uma vez que, dado $x \in L$, a aplicação $y \in N \mapsto(x, y) \in\{x\} \times N$ define uma homotetia com fator de escala $\rho(x)^{2}$. Em particular, as curvaturas seccionais de uma fibra $\{x\} \times N$ satisfazem a seguinte relação

$$
K_{\{x\} \times N}=\frac{1}{\rho^{2}(x)} K_{N}
$$

Exemplo 3. Toda superfície de rotação pode ser vista como um roduto warped, onde as folhas são as diferentes posições da curva perfil e as fibras são os círculos obtidos durante a rotação. Mais precisamente, se $S$ é obtida girando-se uma curva plana $\gamma$ em torno de uma reta em $\mathbb{R}^{3}$ e $\rho$ é a função distância da curva $\rho$ à esta reta, então $S$ é dada por $\gamma \times \rho \mathbb{S}^{1}$

Exemplo 4. Em coordenadas esféricas, a métrica usual de $\mathbb{R}^{3}-\{0\}$ é dada por

$$
d s^{2}=d r^{2}+r^{2}\left(d u^{2}+\sin ^{2} u d v^{2}\right)
$$

Note que, quando $r=1, d s^{2}$ é a métrica da esfera $\mathbb{S}^{2}$. O espaço $\mathbb{R}^{3}-\{0\}$ é difeomorfo a $\mathbb{R}_{+} \times \mathbb{S}^{2}$ através da aplicação

$$
\mathbb{R}_{+} \times \mathbb{S}^{2} \rightarrow t p \in \mathbb{R}^{3}-\{0\} .
$$

Assim, a fórmula para $d s^{2}$ mostra que $\mathbb{R}^{3}-\{0\}$ pode ser identificado com o produto warped $\mathbb{R}_{+} \times{ }_{r} \mathbb{S}^{2}$. As folhas são as semirretas que partem da origem e as fibras são as esferas $\mathbb{S}^{2}(r)$, com $r>0$.

A proposição seguinte, cuja demonstração pode ser encontrada em (NÖLKER, 1996, Corollary 3), caracteriza geometricamente as folhas e fibras de uma variedade produto warped.

Proposição 9. Seja $L \times{ }_{\rho} N$ uma variedade produto warped. Então:

1. As folhas $L \times_{\rho}\{y\}$ são subvariedades totalmente geodésicas;

2. As fibras $\{x\} \times{ }_{\rho} N$ são subvariedades umbílicas com campo vetorial curvatura média dado por $\eta=-\operatorname{grad}\left(\log \rho \circ \pi_{L}\right)$. Além disso, $\eta$ é paralelo ao longo das fibras. 
Corolário 2. Seja $L \times{ }_{\rho} N$ uma variedade produto warped. Então vale a relação

$$
K(\tilde{\sigma})=\frac{1}{\rho^{2}} K_{N}(\sigma)-\|\eta\|^{2}
$$

onde $K$ e $K_{N}$ denotam as curvaturas seccionais de $L \times{ }_{\rho} N$ e $N$, respectivamente, e $\tilde{\sigma}$ é o levantamento vertical de um plano $\sigma$ de $T N$.

Segue do Corolário 2 que se $L \times{ }_{\rho} N$ tem curvatura seccional constante ao longo de alguma fibra, então $N$ tem curvatura seccional constante e $L \times_{\rho} N$ tem curvatura seccional constante ao longo de qualquer fibra.

O resultado a seguir, cuja demonstração pode ser encontrado em (EJIRI, 1983, Lemma 4), complementa o Corolário 2.

Proposição 10. Seja $L \times{ }_{\rho} N$ uma variedade produto warped. Então $L \times{ }_{\rho} N$ tem curvatura seccional constante igual a $c$ se, e somente se, as seguintes condições são simultaneamente satisfeitas:

1. a curvatura seccional de $L$ é constante igual a $c$ e a de $N$ é constante igual a $c \rho^{2}+\|\nabla \rho\|^{2}$;

2. $\operatorname{Hess}_{\rho}()+,c \rho\langle\rangle=$,0 .

A proposição seguinte, cuja demonstração pode ser encontrada em (O’NEILL, 1960, Proposition 7.42), relaciona as curvaturas seccionais de um produto warped ao longo de vetores horizontais e verticais.

Proposição 11. Dada uma variedade produto warped $M=L \times{ }_{\rho} N$, considere os vetores $X, Y$, $U, V \in T_{z} M, \operatorname{com} X$ e $Y$ horizontais e $U$ e $V$ verticais. Então:

1. $K(U, V)=\frac{1}{\rho^{2}}\left(K_{N}(U, V)-|\nabla \rho|^{2}\right)$;

2. $K(X, Y)=K_{L}(X, Y)$;

3. $K(X, U)=-\frac{1}{f} \operatorname{Hess}_{L} \rho(X, X)$.

Corolário 3. Se $I \times{ }_{\rho} N^{n-1}$ é uma variedade produto warped, onde $I$ é um intervalo aberto de $\mathbb{R}$, então $I \times{ }_{\rho} N^{n-1}$ tem curvatura seccional constante igual a $c$ se, e somente se, $N^{n-1}$ tem curvatura seccional constante igual a $k$ e a função $\rho$ satisfaz o sistema de equações diferenciais ordinárias:

$$
\left\{\begin{array}{c}
\frac{\rho^{\prime \prime}}{\rho}=-c, \\
\frac{\left(\rho^{\prime}\right)^{2}-k}{\rho^{2}}=-c .
\end{array}\right.
$$

Finalizaremos esta seção discutindo quando que uma dada G-variedade Riemanniana admite uma estrutura de produto warped. Dizemos que uma variedade Riemanniana $M$, que é de cohomogeneidade k sob a ação de um grupo de Lie $\mathrm{G}$ de isometrias, admite uma estrutura 
adaptada de produto warped se, para todo ponto $\mathrm{p} \in M_{\text {reg }}^{n}$, existe uma vizinhança $\mathrm{U}$ de $\mathrm{p}$ em M que é isométrica a um produto warped $L^{k} \times{ }_{\rho} N$, onde $L^{k}$ é uma componete conexa da parte regular de uma seção de $\mathrm{M}$ e $\mathrm{N}$ é uma variedade Riemanniana isométrica a um aberto da órbita G(p). O teorema seguinte, provado para cohomogeneidade um em (PODESTÀ; SPIRO, 1995, Proposition 2.10) e generalizado para cohomogeneidade k em (GONÇALVES, 2006, Proposição 50), responde a esta questão quando as órbitas da ação são subvariedades umbílicas.

Teorema 20. Seja $M^{n}$ uma $G$-variedade localmente polar completa, onde $G$ é conexo e as órbitas principais são umbílicas e tem cohomogeneidade $\mathrm{k}$, com $1 \leq k \leq n$. Considere a aplicação $\psi: L^{k} \times G\left(x^{n-k}\right) \rightarrow M_{r e g}^{n}$ dada por

$$
\psi(y, g(x))=g(y)
$$

onde $L$ é uma componente conexa da parte regular de uma seção e $x \in L$ é um ponto fixado. Se $\langle,\rangle_{L}$ e $\langle,\rangle_{G(x)}$ denotam as métricas em $L$ e $G(x)$, respectivamente, induzidas pela inclusão em $M^{n}$, então existe uma métrica produto warped de $\langle,\rangle_{L} \mathrm{e}\langle,\rangle_{G(x)}$ que torna $\psi$ uma isometria local sobre $M_{\text {reg }}$ e está normalizada em relação a x.

\subsection{Produtos warped de imersões isométricas}

Nesta seção apresentaremos a noção de produto warped de imersões isométricas, estendendo o caso do produto Riemanniano entre imersões isométricas. Um resultado básico no estudo de decomposições de subvariedades Riemannianas é o seguinte resultado devido a Moore (MOORE, 1971, Main Lemma).

Teorema 21. Seja $f: M^{n} \rightarrow \mathbb{R}^{m}$ uma imersão isométrica de um produto Riemanniano $M=$ $\prod_{i=1}^{n} M_{i}$ conexo cuja segunda forma fundamental $\alpha: T M \times T M \rightarrow T^{\perp} M$ satisfaz

$$
\alpha\left(X_{i}, X_{j}\right)=0
$$

quaisquer $X_{i} \in T L_{i}$ e $X_{j} \in T L_{j}$, onde $L_{1}, \ldots, L_{n}$ são as folheações canônicas induzidas por $M_{1}, \ldots, M_{n}$. Então, existe uma isometria $\phi: N_{1} \times \ldots \times N_{n} \rightarrow \mathbb{R}^{m}$ de um produto Riemanniano de espaços Euclidianos sobre $\mathbb{R}^{m}$ e imersões isoméricas $f_{i}: M_{i} \rightarrow N_{i}, i=1, \ldots, n$, tais que

$$
f=\phi \circ\left(f_{1} \times \ldots \times f_{n}\right) .
$$

Uma imersão isométrica dada por (1.2) é chamada de produto Riemanniano de $f_{1}, \ldots, f_{n}$. Um caso particular importante é dado pelos $m$-cilindros, ou seja, imersões isométricas expressas por $g \times i d: N^{n} \times \mathbb{R}^{m} \rightarrow \mathbb{R}^{n+m+p}=\mathbb{R}^{n+p} \times \mathbb{R}^{m}$, onde $g$ é uma imersão isométrica e $i d: \mathbb{R}^{m} \rightarrow \mathbb{R}^{m}$ é aplicação identidade. Assim, se uma hipersuperfíce do espaço Euclidiano pode ser fatorada como um produto Riemanniano de imersões, então esta tem de ser um cilindro.

A decomposição da subvariedade Euclidiana dada pelo teorema de Moore, através da 
isometria $\phi$, pode ser estendida para as demais formas espaciais. Consideraremos aqui apenas o caso de dois fatores. Uma representação como produto warped de uma forma espacial $\mathbb{Q}_{c}^{n}$ é uma isometria sobre um aberto denso de $\mathbb{Q}_{c}^{n}$ da forma $\phi: V^{n-m} \times_{\sigma} \mathbb{Q}_{\tilde{c}}^{m} \rightarrow \mathbb{Q}_{c}^{n}$, onde $\mathbb{Q}_{\tilde{c}}^{m}$ é um subvariedade esférica de $\mathbb{Q}_{c}^{n}$ e $V^{n-m}$ é um subconjunto aberto da subvariedade totalmente geodéica $\mathbb{Q}_{c}^{n-m} \subset \mathbb{Q}_{c}^{n}$. Uma descrição completa de tais isometrias pode ser encontrada em (NÖLKER, 1996).

Definição 3. Sejam $\phi: V^{n-m} \times_{\sigma} \mathbb{Q}_{\tilde{c}}^{m} \rightarrow \mathbb{Q}_{c}^{n}$ uma representação como produto warped de $\mathbb{Q}_{c}^{n}$ e considere imersões isométricas $f_{1}: L \rightarrow V^{n-m}$ e $f_{2}: N \rightarrow \mathbb{Q}_{\tilde{c}}^{m}$. A imersão isométrica $f=$ $\phi \circ\left(f_{1} \times f_{2}\right): L \times{ }_{\rho} N \rightarrow \mathbb{Q}_{c}^{n}$ onde $\rho=\sigma \circ f_{1}$ é chamada o produto warped de $f_{1}$ e $f_{2}$ determinado por $\phi$.

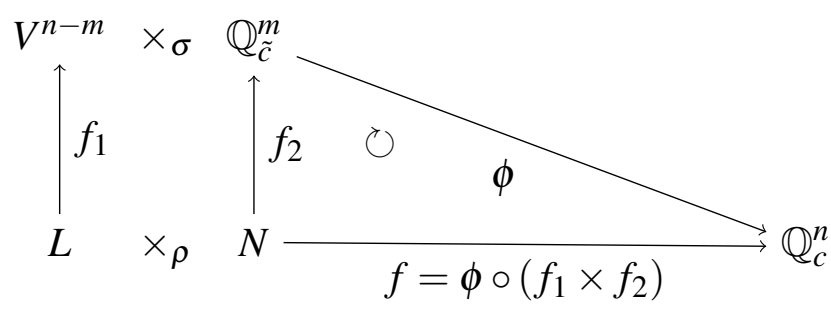

As subvariedades apresentadas a seguir são exemplos naturais de produto warped de imersões isométricas. Além disso, são exemplos das subvariedades que classificaremos adiante.

Exemplo 5. Seja $f=\phi \circ\left(f_{1} \times f_{2}\right): L \times{ }_{\rho} N \rightarrow \mathbb{Q}_{c}^{n}$ a imersão isométrica dada como produto warped de $f_{1}$ e $f_{2}$, determinada pela representação $\phi: V^{n-m} \times_{\sigma} \mathbb{Q}_{\tilde{c}}^{m} \rightarrow \mathbb{Q}_{c}^{n}$ de $\mathbb{Q}_{c}^{n}$. Quando $f_{2}: N \rightarrow \mathbb{Q}_{\tilde{c}}^{m}$ é uma isometria e $L \times_{\rho} N$ não é um produto Riemanniano, diremos que $f$ é uma subvariedade rotacional com curva perfil $f_{1}$.

Exemplo 6. Considere a representação $\phi: \mathbb{R}_{+} \times{ }_{\sigma} \mathbb{S}^{n+1} \rightarrow \mathbb{R}^{n+2}$ dada pelas coordenadas esféricas de $\mathbb{R}^{n+2}$. Dada uma subvariedade $f: N^{m} \rightarrow \mathbb{S}^{n+1}$, o produto warped $\phi \circ(i d \times f): \mathbb{R}_{+} \times{ }_{\rho} N^{m} \rightarrow$ $\mathbb{R}^{n+2}$, dado por $(t, p) \mapsto t f(p)$, é uma parametrização isométrica global da subvariedade que generaliza a noção de cone em $\mathbb{R}^{3}$, e é chamada de cone generalizado sobre $\mathrm{f}$.

Com a generalização das coordenadas esféricas para as formas espaciais $\mathbb{S}_{c}^{n+2}$ e $\mathbb{H}_{c}^{n+2}$, a noção de cone se estende naturalmente para estes ambientes. Assim, se $\phi: V \times_{\sigma} \mathbb{Q}_{\tilde{c}}^{n+1} \rightarrow \mathbb{Q}_{c}^{n+2}$ é uma representação como produto warped de $\mathbb{Q}_{c}^{n+2}$, onde $V$ é um aberto de $\mathbb{Q}_{c}^{1}$ e $f: N^{m} \rightarrow \mathbb{Q}_{\tilde{c}}^{n+1}$ é uma imersão isométrica, tem-se que o cone sobre $f$ é parametrizado por

$$
(t, p) \in V \times{ }_{\sigma} N^{m} \mapsto \phi(t, f(p)) \in \mathbb{Q}_{c}^{n+2}
$$

Uma propriedade geométrica que caracteriza os cones de $\mathbb{R}^{3}$ é que estes são formados por semirretas a partir da origem que passam por uma curva da esfera. De modo mais geral, dados uma representação como produto warped $\phi: V^{k} \times_{\sigma} \mathbb{Q}_{\tilde{c}}^{n-k+2} \rightarrow \mathbb{Q}_{c}^{n+2}$ da forma espacial $\mathbb{Q}_{c}^{n+2}$ e uma 
imersão $f: N^{n-k} \rightarrow \mathbb{Q}_{\tilde{c}}^{n-k+2}$, considere a subvariedade esférica $\Omega^{n-k+1}=\phi\left(\{\bar{x}\} \times \mathbb{Q}_{\tilde{c}}^{n-k+2}\right)$, para algum $\bar{x} \in V^{k}$. Então a imagem da parametrização $\phi \circ\left(i d_{V} \times f\right): V^{k} \times{ }_{\sigma} N^{n-k} \rightarrow \mathbb{Q}_{c}^{n+2}$ é a união de subvariedades geodésicas 1-dimensionais ortogonais a $\Omega$ e que passam por uma subvariedade de $\Omega^{n-k+2}$. Uma subvariedade da forma $\phi \circ\left(i d_{V} \times f\right)$ será chamada de o cone sobre $f$ segundo a representação $\phi$. Assim, dado o produto warped $f=\phi \circ\left(f_{1} \times f_{2}\right): L^{k} \times{ }_{\rho} N^{n-k} \rightarrow \mathbb{Q}_{c}^{n+2}$ determinado por $\phi: V^{k} \times_{\sigma} \mathbb{Q}_{\tilde{c}}^{n-k+2} \rightarrow \mathbb{Q}_{c}^{n+2}$, se $f_{1}$ é uma isometria sobre a imagem então $f$ é parte do cone sobre $f_{2}$ segundo $\phi$. Em codimensão 1, qualquer produto warped de imersões isométricas é dado em termos dos exemplos anteriores (cf.(DAJCZER; TOJEIRO, 2004)). Em codimensão 2, discutiremos na seção seguinte que uma terceira possibilidade pode ocorrer, o caso em que $f_{1}$ e $f_{2}$ são hipersuperfícies.

A proposição a seguir relaciona a segunda forma fundamental do produto warped de duas imersões isométricas com as dos fatores.

Proposição 12. Sejam $M=L \times{ }_{\rho} N$ e $\tilde{M}=\tilde{L} \times \tilde{\rho} \tilde{N}$ variedades produto warped e sejam $F: L \rightarrow \tilde{L}$ e $G: M \rightarrow \tilde{M}$ imersões isoméricas, $\operatorname{com} \rho=\tilde{\rho} \circ F$. Então $f=F \times G: M \rightarrow \tilde{M}$ é uma imersão isométrica e, para cada ponto $z=(x, y) \in M$, valem as seguintes propriedades:

1. $\pi_{L_{*}} f_{*} T_{z} M=F_{*} T_{x} L, \pi_{L_{*}} T_{z}^{\perp} M=T_{x}^{\perp} L, \pi_{\tilde{N}_{*}} f_{*} T_{z} M=G_{*} T_{y} N, \quad \pi_{\tilde{N}_{*}} T_{z}^{\perp} M=T_{y}^{\perp} N$;

2. $(\nabla \tilde{\rho}(F(x)))_{T_{x}^{\perp} L}=\nabla \tilde{\rho}(F(x))-F_{*} \nabla \rho(x)$;

3. As segunda formas fundamentais de $F, G$ e $f$ estão relacionadas por

$$
\begin{gathered}
\pi_{\tilde{N}_{*}} \alpha^{f}(X, Y)=\alpha^{G}\left(\pi_{N_{*}} X, \pi_{N_{*}} Y\right), \\
\pi_{\tilde{L}_{*}} \alpha^{f}(X, Y)=\alpha^{F}\left(\pi_{L_{*}} X, \pi_{L_{*}} Y\right)-\rho(x)\left\langle\pi_{N_{*}} X, \pi_{N_{*}} Y\right\rangle\left(\nabla_{\tilde{\rho}}(F(x))\right)_{T_{x}^{\perp} L},
\end{gathered}
$$

para quaisquer $X, Y \in T M$.

Definição 4. Sejam $M$ uma variedade diferenciável e $T M=E_{1} \oplus E_{2}$ uma decomposição em dois subfibrado de $T M$, denotada por $\varepsilon=\left(E_{1}, E_{2}\right)$. A família $\varepsilon$ é chamada uma 2-rede se os subfibrados de $\varepsilon$ são integráveis.

Se $M$ é uma variedade Riemanniana e $E_{1}$ e $E_{2}$ são ortogonais, então $\varepsilon$ é dita uma 2-rede ortogonal.

Exemplo 7. Seja $M=L \times N$ uma variedade diferenciável produto. Neste caso, $\varepsilon=\{\mathscr{H}, \mathscr{V}\}$, formada pelos subfibrados horizontal e vertical, é uma 2-rede, chamada a rede produto.

Definição 5. Sejam $M$ uma variedade Riemanniana e $\varepsilon=\left(E_{1}, E_{2}\right)$ uma rede ortogonal em $M$. Diz-se que $\varepsilon$ é uma rede produto warped se $E_{1}$ é um subfibrado vetorial totalmente geodésico e $E_{2}$ é um subfibrado vetorial esférico. 
Seja $f: M^{n+l}=L^{l} \times_{\rho} N^{n} \rightarrow \mathbb{Q}_{c}^{m}$ uma imersão isométrica de um produto warped de variedades Riemannianas e seja $\varepsilon=(\mathscr{H}, \mathscr{V})$ a rede produto. Dizemos que a segunda forma fundamental de $f$ é adaptada à rede produto $\varepsilon$ se satisfaz

$$
\alpha(X, V)=0
$$

para quaisquer $X \in \mathscr{H}$ e $V \in \mathscr{V}$.

Quando uma imersão isométrica é um produto warped de imersões determinado por alguma representação, segue da Propoposição 12 que a segunda forma fundamental da imersão é adaptada à rede produto do domínio. O teorema a seguir, devido a Nolker (NÖLKER, 1996, Theorem 7), é um resultado clássico e estabelece a recíproca da Proposição 12.

Teorema 22. Seja $f: L \times_{\rho} N \rightarrow \mathbb{Q}_{c}^{m}$ uma imersão isométrica de um produto warped conexo cuja segunda forma fundamental é adaptada a rede produto de $L \times_{\rho} N$. Fixado um ponto $(\bar{x}, \bar{y})$ $\in L \times{ }_{\rho} N$, com $\rho(\bar{x})=1$, sejam $f_{1}: L \rightarrow \mathbb{Q}_{c}^{m}$ e $f_{2}: N \rightarrow \mathbb{Q}_{c}^{m}$ as imersões isométricas dadas por $f_{1}(x)=f(x, \bar{y})$ e $f_{2}(y)=f(\bar{x}, y)$. Então $f$ é o produto warped de $f_{1}$ e $f_{2}$ determinado pela representação como produto warped de $\mathbb{Q}_{c}^{m}$ a partir do fecho esférico de $f_{2}$ e do ponto $f(\bar{x}, \bar{y})$.

Dado uma imersão isométrica $f: L \times{ }_{\rho} N \rightarrow \mathbb{Q}_{c}^{m}$ de um produto warped, considere o tensor tipo-curvatura $\mathrm{C}$ dado por

$$
\begin{gathered}
C\left(E_{1}, E_{2}, E_{3}, E_{4}\right):=\left\langle R\left(E_{1}, E_{2}\right) E_{3}, E_{4}\right\rangle-c\left\langle\left(E_{1} \wedge E_{2}\right) E_{3}, E_{4}\right\rangle= \\
\left\langle\alpha\left(E_{1}, E_{4}\right), \alpha\left(E_{2}, E_{3}\right)\right\rangle-\left\langle\alpha\left(E_{1}, E_{3}\right), \alpha\left(E_{2}, E_{4}\right)\right\rangle
\end{gathered}
$$

Proposição 13. O tensor tipo-curvatura $\mathrm{C}$ satisfaz as seguintes propriedades:

$$
\begin{gathered}
C(X, V, W, Y)=\langle V, W\rangle\left\langle\nabla_{X} \eta-\langle X, \eta\rangle \eta-c X, Y\right\rangle, \\
C(X, Y, V, Z)=0 \\
C(X, Y, V, W)=0 \\
C(X, U, V, W)=0 .
\end{gathered}
$$

\subsection{Teorema de classificação de Dajczer-Tojeiro}

Nesta seção iremos recapitular um teorema de classificação local de imersões isométricas $f: L^{p} \times{ }_{\rho} N^{n} \rightarrow \mathbb{Q}_{c}^{p+n+2}$, devido a Dajczer e Tojeiro (DAJCZER; TOJEIRO, 2004), sob a hipótese de que $n \geq 3$ e que $L^{p} \times{ }_{\rho} N^{n}$ não tenha pontos de mesma curvatura seccional constante c do espaço ambiente. Mais precisamente, os autores provam o seguinte resultado 
Teorema 23 ((DAJCZER; TOJEIRO, 2004, Theorem 14)). Seja $N^{n+p}=L^{p} \times{ }_{\rho} M^{n}$ uma variedade Riemanniana produto warped, com $n \geq 3$ e livre de pontos com curvatura seccional constante igual a $c$. Dado uma imersão isométrica $f: N^{n+p} \rightarrow \mathbb{Q}_{c}^{n+p+2}$, existe um subconjunto aberto e denso de $N^{n+p}$ tal que cada ponto desse aberto pertence a uma vizinhança produto $U=$ $L_{0}^{p} \times N_{0}^{n} \subset L^{p} \times{ }_{\rho} N^{n}$, de modo que uma das seguinte possibilidades ocorre:

1. $\left.f\right|_{U}$ é um produto warped de imersões isométricas em relação a uma representação de produto warped $\psi: V^{p+k_{1}} \times_{\sigma} \mathbb{Q}_{\tilde{c}}^{n+k_{2}} \rightarrow \mathbb{Q}_{c}^{p+n+2}, \operatorname{com} k_{1}+k_{2}=2$.

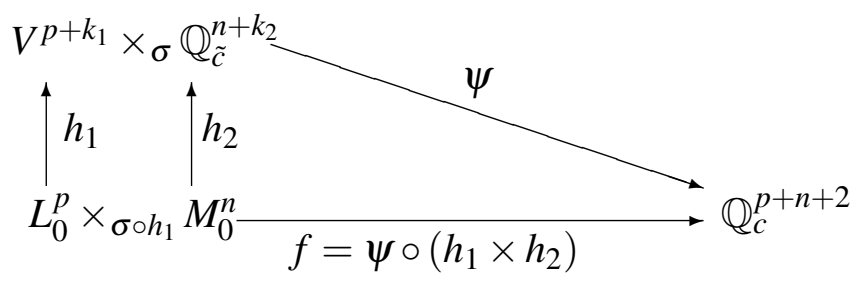

2. $\left.f\right|_{U}$ é uma composição $H \circ g$ de imersões isométricas, onde $g$ é um produto warped de imersões isométricas $g=\psi \circ\left(h_{1} \times h_{2}\right)$, determinada por uma representação de produto warped $\psi: V^{p+k_{1}} \times_{\sigma} \mathbb{Q}_{\tilde{c}}^{n+k_{2}} \rightarrow \mathbb{Q}_{c}^{p+n+1}$, com $k_{1}+k_{2}=1$, e $H: W \rightarrow \mathbb{Q}_{c}^{n+p+2}$ é uma imersão isométrica de um subconjunto aberto $W \subset \mathbb{Q}_{c}^{n+p+1}, \operatorname{com} g(U) \subset W$.

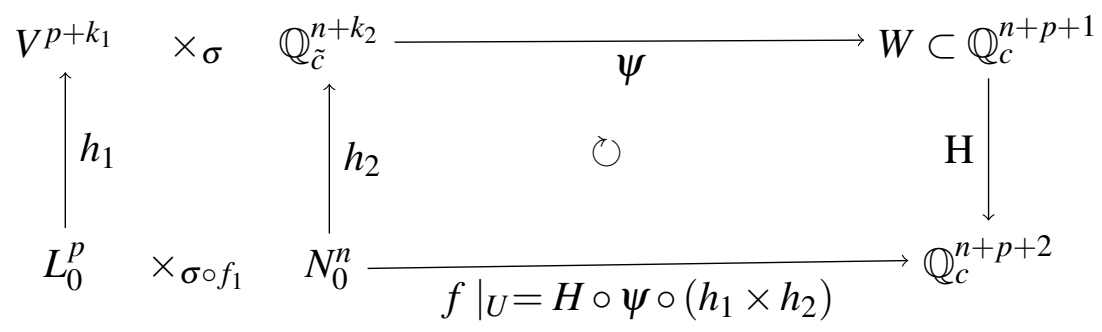

3. Existem intervalos abertos $I, J \subset \mathbb{R}$ tais que $L_{0}^{p}, M_{0}^{n}$ e $U$ decompõe-se como $L_{0}^{p}=L_{0}^{p-1} \times \rho_{1}$ $I, M_{0}^{n}=J \times \rho_{2} M_{0}^{n-1} \mathrm{e}$

$$
U=L_{0}^{p-1} \times \rho_{1}\left(\left(I \times \rho_{3} J\right) \times \bar{\rho} M_{0}^{n-1}\right),
$$

onde $\rho_{1} \in C^{\infty}\left(L_{0}^{n-1}\right), \rho_{2} \in C^{\infty}(J), \rho_{3} \in C^{\infty}(I)$ e $\bar{\rho} \in C^{\infty}(I \times J)$ satisfazem

$$
\rho=\left(\rho_{1} \circ \pi_{L_{0}^{p-1}}\right)\left(\rho_{3} \circ \pi_{I}\right) \text { e } \bar{\rho}=\left(\rho_{3} \circ \pi_{I}\right)\left(\rho_{2} \circ \pi_{J}\right),
$$

e existem representações de produto warped

$$
\psi_{1}: V^{p-1} \times_{\sigma_{1}} \mathbb{Q}_{c}^{n+3} \rightarrow \mathbb{Q}_{c}^{p+n+2} \text { e } \psi_{2}: W^{4} \times_{\sigma_{2}} \mathbb{Q}_{c}^{n-1} \rightarrow \mathbb{Q}_{c}^{n+3},
$$

uma imersão isométrica $g: I \times \rho_{3} J \rightarrow W^{4}$ e isometrias $i_{1}: L_{0}^{p-1} \rightarrow W^{p-1} \subset V^{p-1} \subset \mathbb{Q}_{c}^{p-1}$ e $i_{2}: M^{n-1} \rightarrow W^{n-1} \subset \mathbb{Q}_{c}^{n-1}$ sobre subconjuntos abertos tais que

$$
\left.f\right|_{U}=\psi_{1} \circ\left(i_{1} \times\left(\psi_{2} \circ\left(g \times i_{2}\right)\right)\right),
$$


$\rho=\sigma_{2} \circ g$ e $\rho_{1}=\sigma_{1} \circ i_{1}$. Além disso, $L_{0}^{p}$ tem curvatura seccional constante $c$, se $p \geq 2$.

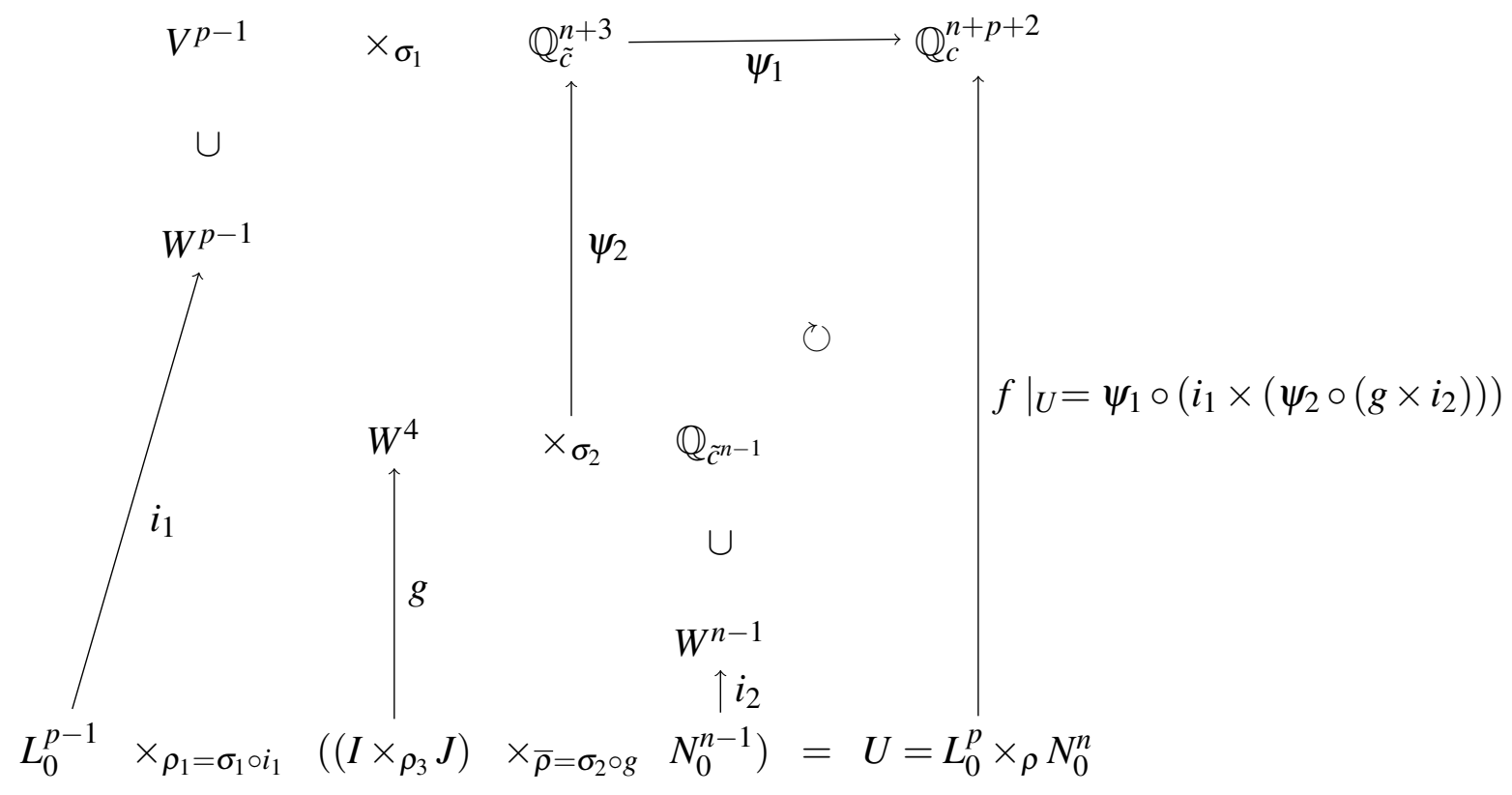





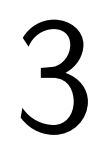

\section{SUBVARIEDADES DE CODIMENSÃO DOIS EM FORMAS ESPACIAIS}

\subsection{Resultados auxiliares}

Nesta seção provaremos alguns resultados preliminares que serão usados nas seções seguintes, onde obteremos os teoremas principais desta tese.

Dada uma imersão isométrica $f: L^{p} \times{ }_{\rho} N^{n} \rightarrow \mathbb{Q}_{c}^{l}$ e fixado um ponto $z \in L^{p} \times{ }_{\rho} N^{n}$, considere o conjunto

$$
\alpha_{f}\left(\mathscr{H}_{z}, \mathscr{V}_{z}\right)=\operatorname{span}\left\{\alpha_{f}(X, V): X \in \mathscr{H}_{z} \text { e } V \in \mathscr{V}_{z}\right\}
$$

Dizemos que a imersão $f$ no ponto $z$ é do tipo:

(A) se $\operatorname{dim} \alpha_{f}\left(\mathscr{H}_{z}, \mathscr{V}_{z}\right)=0$,

(B) $\operatorname{se} \operatorname{dim} \alpha_{f}\left(\mathscr{H}_{z}, \mathscr{V}_{z}\right)=1$,

(C) $\operatorname{se} \operatorname{dim} \alpha_{f}\left(\mathscr{H}_{z}, \mathscr{V}_{z}\right) \geq 2$.

Note que as imersões isométricas $f: L^{p} \times{ }_{\rho} N^{n} \rightarrow \mathbb{Q}_{c}^{l}$ do tipo A são justamente aquelas do teorema de decomposição de Nölker (NÖLKER, 1996), i.e., $\alpha_{f}(X, V)=0$ para quaisquer $X \in \mathscr{H}_{z}$ e $V \in \mathscr{V}_{z}$. Além disso, imersões isométricas $f: L^{p} \times_{\rho} N^{n} \rightarrow \mathbb{Q}_{c}^{p+n+2}$ do tipo C, com $n \geq 3$, têm curvatura seccional constante igual a $c$ (cf. (DAJCZER; TOJEIRO, 2004, Proposition 36)). Dessa forma, o caso interessante a ser estudado é o das imersões do tipo B.

Dada uma imersão isométrica $f: L^{p} \times{ }_{\rho} N^{n} \rightarrow \mathbb{Q}_{c}^{l}$, fixemos um ponto $z \in L^{p} \times{ }_{\rho} N^{n}$. Dado um vetor não-nulo $X \in \mathscr{H}_{z}$, considere a aplicação linear $B_{X}: \mathscr{V}_{z} \rightarrow T_{z}\left(L^{p} \times_{\rho} N^{n}\right)^{\perp}$ dada por

$$
B_{X}(V)=\alpha_{f}(X, V),
$$


para todo $V \in \mathscr{V}_{z}$.

Proposição 14 ((DAJCZER; TOJEIRO, 2004, Lemma 20)). Se uma imersão isométrica $f$ : $L^{p} \times{ }_{\rho} N^{n} \rightarrow \mathbb{Q}_{c}^{l}$ não é do tipo A no ponto $z \in L^{p} \times_{\rho} N^{n}$ e, para cada $X \in \mathscr{H}_{z}$, tem-se $\operatorname{rank} B_{X} \leq 1$ então, a menos de sinal, existe um único vetor unitário $e \in \mathscr{V}_{z}$ tal que

$$
\alpha_{f}(X, V)=\langle V, e\rangle \alpha_{f}(X, e)
$$

$\mathrm{e}$

$$
\alpha_{f}(V, W)-\langle V, e\rangle\langle W, e\rangle \alpha_{f}(e, e) \perp \alpha_{f}\left(\mathscr{H}_{z}, \mathscr{V}_{z}\right)
$$

para quaisquer $X \in \mathscr{H}_{z}$ e $V, W \in \mathscr{V}_{z}$.

No caso em que a codimensão é 2, obtemos o seguinte

Lema 1. Seja $f: L^{p} \times{ }_{\rho} N^{n} \rightarrow \mathbb{Q}_{c}^{p+n+2}$ uma imersão isométrica do tipo B no ponto $z \in L^{p} \times{ }_{\rho} N^{n}$, e que não seja do tipo $\mathrm{A}$ em $z$. Então, a menos de sinal, existe um único vetor unitário $e \in \mathscr{V}_{z}$ tal que

$$
\alpha_{f}(V, W)=\langle V, e\rangle\langle W, e\rangle \alpha_{f}(e, e)
$$

para quaisquer $V, W \in \mathscr{V}_{z}$.

Demonstração. Como $f$ é do tipo $B$ no ponto $z$, temos

$$
\operatorname{rank} B_{X} \leq \operatorname{dim} \alpha_{f}\left(\mathscr{H}_{z}, \mathscr{V}_{z}\right)=1
$$

para todo $X \in \mathscr{H}_{z}$. Assim, segue da Proposição 14 que existe um único vetor unitário $e \in \mathscr{V}_{z}$ para o qual vale (3.3). Considere então a aplicação linear $\phi: \mathscr{V}_{z} \times \mathscr{V}_{z} \rightarrow T_{z}\left(L^{p} \times{ }_{\rho} N^{n}\right)^{\perp}$ dada por

$$
\phi(V, W)=\alpha_{f}(V, W)-\langle V, e\rangle\langle W, e\rangle \alpha_{f}(e, e) .
$$

Provar (3.4) é equivalente a mostrar que $\operatorname{rank} \phi=0$. Em virtude de (3.3) temos que rank $\phi \leq 1$. Observe, inicialmente, que se $\operatorname{dim} \mathscr{V}_{z}=1$ a conclusão segue diretamente. Assim, podemos assumir que $\operatorname{dim} \mathscr{V}_{z} \geq 2$. Como $\alpha_{f}(e, e) \neq 0$, basta mostrar que $\operatorname{dim} \alpha_{f}\left(\mathscr{V}_{z}, \mathscr{V}_{z}\right)=1$. De fato, suponha por absurdo que $\operatorname{dim} \alpha_{f}\left(\mathscr{V}_{z}, \mathscr{V}_{z}\right)=2$. Assim, existem vetores $V_{0}, W_{0} \in \mathscr{V}_{z}$, onde pelo menos um deles pertence ao subespaço $\{e\}^{\perp}$, tais que

$$
\alpha_{f}\left(\mathscr{V}_{z}, \mathscr{V}_{z}\right)=\operatorname{span}\left\{\alpha_{f}(e, e), \alpha_{f}\left(V_{0}, W_{0}\right)\right\}
$$

Isso implica que

$$
\phi(V, W)=a \alpha_{f}\left(V_{0}, W_{0}\right)+(b-\langle V, e\rangle\langle W, e\rangle) \alpha_{f}(e, e),
$$

para quaisquer $V, W \in \mathscr{V}_{z}$, onde $a$ e $b$ são constantes que dependem, possivelmente, de $V$ e $W$. Disso decorre, em particular, que rank $\phi=2$, o que é uma contradição. 
Como consequência do Lema 1, obtemos o seguinte resultado quando a variedade $L$ tem dimensão 1 .

Proposição 15. Se uma imersão isométrica $f: L \times_{\rho} N^{n-1} \rightarrow \mathbb{Q}_{c}^{n+2}$ é do tipo B ou C num ponto $z \in L \times{ }_{\rho} N^{n-1}$, então a segunda forma fundamental de f satisfaz:

$$
\alpha_{f}(E, V)=0
$$

para quaisquer $E \in T_{Z}\left(L \times{ }_{\rho} N^{n-1}\right)$ e $V \in \operatorname{ker} B_{X}$, onde $B_{X}$ é a aplicação linear dada em (3.1).

Demonstração. Suponha, inicialmente, que f é do tipo B no ponto $z \in L \times{ }_{\rho} N^{n-1}$. Assim, para todo $X \in \mathscr{H}_{z}$, tem-se

$$
\operatorname{rank} B_{X} \leq \operatorname{dim} \alpha_{f}\left(\mathscr{H}_{z}, \mathscr{V}_{z}\right)=1
$$

logo a Proposição 14 se aplica neste caso. Da equação (3.2) segue que $\operatorname{ker} B_{X}=\{e\}^{\perp}$. Em particular, isso mostra que a igualdade (3.6) é satisfeita para todo $E \in \mathscr{H}_{z}$. Por outro lado, segue do Lema 1 que

$$
\alpha_{f}(V, W)=\langle v, e\rangle\langle W, e\rangle \alpha_{f}(e, e)
$$

para quaisquer $V, W \in \mathscr{V}_{z}, \operatorname{logo}$ a igualdade (3.6) vale também para todo $E \in \mathscr{V}_{z}$. Suponha agora que f é do tipo C no ponto $z \in L \times{ }_{\rho} N^{n-1}$. Assim, para todo $X \in \mathscr{H}_{z}$, temos que

$$
\operatorname{rank} B_{X} \leq \operatorname{dim} \alpha_{f}\left(\mathscr{H}_{z}, \mathscr{V}_{z}\right)=2
$$

Resta considerar o caso em que rank $B_{x}=2$, ou seja, $B_{X}\left(\mathscr{V}_{z}\right)=T_{z}\left(I \times{ }_{\rho} N^{n-1}\right)^{\perp}$. Neste caso, a igualdade (3.6) é equivalente a

$$
\left\langle\alpha_{f}(E, V), \alpha_{f}(X, W)\right\rangle=0
$$

para quaisquer $V \in \operatorname{ker}_{X}, W \in \mathscr{V}_{z}$ e $E \in T_{z}\left(I \times{ }_{\rho} N^{n-1}\right)$. Porém, isto segue de (2.10) para todo $E \in \mathscr{H}_{z}$ e de (2.11) para $E \in \mathscr{V}_{z}$.

Consideremos uma $G$-variedade Riemanniana completa $M^{n}$ com cohomogeneidade $1 \mathrm{e}$ órbitas principais umbílicas, onde $G \subset I s o(M)$ é um subgrupo fechado. Dado um ponto $p \in M_{\text {reg }}$, segue do Teorema 20 que existe uma vizinhança $\Gamma$ de p em $M$ isométrica a um produto warped $I \times{ }_{\rho} N^{n-1}$, onde $I \subset \mathbb{R}$ é um intervalo aberto e $N^{n-1}$ é um aberto da órbita $G(p)$.

Com a notação acima, temos a seguinte

Proposição 16. Se $f: I \times{ }_{\rho} N^{n-1} \rightarrow \mathbb{Q}_{c}^{n+2}$ é uma imersão isométrica tipo $B$, então $I \times{ }_{\rho} N^{n-1}$ tem curvatura seccional constante igual a $c$.

Demonstração. Como $f$ é do tipo $B$ em todo ponto $z \in I \times{ }_{\rho} N^{n-1}$, segue da Proposição 15 que a segunda forma fundamental $\alpha_{f}$ de f satisfaz

$$
\alpha_{f}(E, V)=0
$$


para quaisquer $E \in T_{z}\left(I \times{ }_{\rho} N^{n-1}\right)$ e $V \in \operatorname{Ker} B_{X}$, onde $B_{X}$ é dado em (3.1). Dados $Y \in \mathscr{H}_{z}$ e $V \in \mathscr{V}_{z}, \operatorname{com}\langle V, Y\rangle=0$, segue da equação de Gauss e do item (3) da Proposição 11 que:

$$
c=-\frac{\rho^{\prime \prime}(t)}{\rho(t)},
$$

para todo $t \in I$. Por outro lado, como $\operatorname{rank} B_{X}=1$, tem-se $\operatorname{dim} \operatorname{ker} B_{X}=n-2$. Escolha uma base $\left\{e_{2}, \ldots, e_{n-1}\right\}$ para $\operatorname{ker} B_{X}=\{e\}^{\perp}$ de modo que $\left\{e_{1}, e_{2}, \ldots, e_{n-1}\right\}$ seja base de $\mathscr{V}_{z}$, onde $e_{1}=e$. Note que, em virtude do Lema 15 , temos que

$$
\alpha_{f}\left(e_{i}, e_{j}\right)=0
$$

para todo $1 \leq i \leq n-1$ e $2 \leq j \leq n-1$. Usando a equação de Gauss aplicada á base $\left\{e_{1}, e_{2}, \ldots, e_{n-1}\right\}$ de $\mathscr{V}_{z}$, concluimos que as curvaturas seccionais de $I \times{ }_{\rho} N^{n-1}$ satisfazem

$$
K\left(e_{i}, e_{j}\right)=c+\left\langle\alpha_{f}\left(e_{i}, e_{i}\right), \alpha\left(e_{j}, e_{j}\right)\right\rangle-\left\|\alpha_{f}\left(e_{i}, e_{j}\right)\right\|^{2}=c,
$$

para quaisquer $1 \leq i \leq n-1$ e $2 \leq j \leq n-1$. Assim, em virtude da Proposição 8, segue que as órbitas principais têm curvatura seccional constante. Em particular, $N^{n-1}$ tem curvatura seccional constante. Mais precisamente, em virtude da Proposição 11, item (i), as curvaturas seccionais $K$ do produto warped $I \times{ }_{\rho} N^{n-1}$ e as curvaturas seccionais $K_{N}$ de $N$ satisfazem

$$
K_{N}\left(e_{i}, e_{j}\right)=\rho^{2} K\left(e_{i}, e_{j}\right)+\left|\rho^{\prime}\right|^{2}=c \rho^{2}+\left(\rho^{\prime}\right)^{2},
$$

para quaisquer $1 \leq i \leq n-1$ e $2 \leq j \leq n-1$. Como $\left\{e_{1}, \ldots, e_{n-1}\right\}$ é base para $\mathscr{V}_{z}$, segue que $N^{n-1}$ tem curvatura seccional constante igual a $c(\rho(t))^{2}+\left(\rho^{\prime}(t)\right)^{2}$. De (3.11) e (3.12) segue que a função warping $\rho$ satisfaz o sistema

$$
\left\{\begin{array}{c}
\frac{\rho^{\prime \prime}}{\rho}=-c, \\
\frac{\left(\rho^{\prime}\right)^{2}-K_{N}}{\rho^{2}}=-c,
\end{array}\right.
$$

implicando, em virtude do Corolário 3, que $I \times{ }_{\rho} N^{n-1}$ tem curvatura seccional constante igual a $c$.

\subsection{Resultado local}

Nesta seção provaremos que, embora o produto warped $I \times{ }_{\rho} G(p)^{n-1}$ tenha pontos com curvatura seccional constante igual a curvatura do ambiente, ainda assim a imersão isométrica possui a segunda forma fundamental adaptada à rede produto de $I \times{ }_{\rho} G(p)^{n-1}$.

Para isso, faremos uso de alguns resultados preliminares.

Proposição 17 ((DAJCZER; TOJEIRO, 2004, Lemma 21)). Seja $f: L^{p} \times{ }_{\rho} N^{n} \rightarrow \mathbb{Q}_{c}^{l}$ uma imersão isométrica do tipo B num ponto $z \in L^{p} \times{ }_{\rho} N^{n}$. Então, a menos de sinal, existem únicos 
vetores unitários $X \in \mathscr{H}_{z}, e \in \mathscr{V}_{z}$ e $\xi \in T_{z}\left(L^{p} \times{ }_{\rho} N^{n}\right)$, e constantes $\beta, \lambda, \gamma \in \mathbb{R}$, com $\lambda \neq 0$, tais que

$$
\begin{gathered}
\left\langle\alpha_{f}(Y, Z), \xi\right\rangle=\beta\langle Y, X\rangle\langle Z, X\rangle, \\
\alpha_{f}(Y, V)=\lambda\langle Y, X\rangle\langle V, e\rangle \alpha(Y, e) \xi, \\
\left\langle\alpha_{f}(V, W), \xi\right\rangle=\gamma\langle V, e\rangle\langle W, e\rangle \\
\left\langle\tilde{P} \alpha_{f}(Y, Z), \tilde{P} \alpha_{f}(V, W)-\langle V, W\rangle \tilde{P} \alpha_{f}(e, e)\right\rangle=\left(\beta \gamma-\lambda^{2}\right)\langle Y, X\rangle\langle Z, X\rangle\langle P V, P W\rangle,
\end{gathered}
$$

onde $\tilde{P}: T_{z}^{\perp}\left(L^{p} \times{ }_{\rho} N^{n}\right) \rightarrow T_{z}^{\perp}\left(L^{p} \times{ }_{\rho} N^{n}\right)$ e $P: \mathscr{V}_{z} \rightarrow \mathscr{V}_{z}$ são as projeções ortogonais sobre os subespaços $\{\xi\}^{\perp} \subset T_{z}^{\perp}\left(L^{p} \times{ }_{\rho} N^{n}\right)$ e $\{e\}^{\perp} \subset \mathscr{V}_{z}$, respectivamente.

As equações (3.13), (3.14) e (3.15) são equivalentes a:

$$
A_{\xi} Y=\langle Y, X\rangle(\beta X+\lambda e), \quad A_{\xi} V=\langle V, e\rangle(\lambda X+\gamma e),
$$

e

$$
\tilde{P} \alpha_{f}(Y, V)=0 \text {. }
$$

Em particular, segue de (3.17) que o posto de $A_{\xi}$, no ponto $z$, é igual a 1 ou 2 , dependendo se $\beta \gamma-\lambda^{2}$ é igual a zero ou diferente de zero, respectivamente. De acordo com isso, dizemos que $f$ é do tipo $B_{1}$ ou do tipo $B_{2}$ no ponto $z$.

Seja $f: L^{p} \times_{\rho} N^{n} \rightarrow \mathbb{Q}_{c}^{p+n+2}$ uma imersão isométrica do tipo B em um ponto $z \in$ $L^{p} \times{ }_{\rho} N^{n}$, e considere $X, e, \xi, \beta, \gamma$ e $\gamma$ dados pela Proposição 17. Fixemos um vetor unitário $\tilde{\xi} \in T_{z}^{\perp}\left(L^{p} \times{ }_{\rho} N^{n}\right)$, ortogonal a $\xi$, e consideremos as formas bilineares simétricas

$$
\tilde{\beta}: \mathscr{H}_{z} \times \mathscr{H}_{z} \rightarrow \mathbb{R} \quad \text { e } \quad \tilde{\gamma}: \mathscr{V}_{z} \times \mathscr{V}_{z} \rightarrow \mathbb{R}
$$

dadas por

$$
\tilde{\beta}(Y, Z)=\langle\alpha(Y, Z), \tilde{\xi}\rangle \quad \text { e } \quad \tilde{\gamma}(V, W)=\langle\alpha(V, W), \tilde{\xi}\rangle
$$

Considere também

$$
\tilde{\beta}_{0}=\tilde{\beta}(X, X), \quad \tilde{\gamma}_{0}=\tilde{\gamma}(e, e) \quad \text { e } \quad \tilde{\delta}_{0}=\tilde{\beta}_{0} \tilde{\gamma}_{0}+\beta \gamma-\lambda^{2}
$$

Proposição 18 ((DAJCZER; TOJEIRO, 2004, Proposition 25)). Seja $f: L^{p} \times{ }_{\rho} N^{n} \rightarrow \mathbb{Q}_{c}^{p+n+2}$ uma imersão isométrica do tipo B num ponto $z \in L^{p} \times{ }_{\rho} N^{n}$. Então:

(i) Se $f$ é do tipo $B_{1}$ no ponto $z$, então $\tilde{\gamma}(V, W)=\langle V, W\rangle \tilde{\gamma}_{0}$ ou $\tilde{\beta}=0$. 
(ii) Se $n \geq 2$ e $f$ é do tipo $B_{2}$ em z, então

$$
\tilde{\beta}(Y, Z)=\langle Y, X\rangle\langle Z, X\rangle \tilde{\beta}_{0}, \quad \tilde{\beta}_{0} \neq 0
$$

e

$$
\tilde{\beta}_{0} \tilde{\gamma}(V, W)=\langle V, W\rangle \tilde{\delta}_{0}-\left(\beta \gamma-\lambda^{2}\right)\langle V, e\rangle\langle W, e\rangle
$$

Com a mesma notação da Proposição 16, podemos provar o principal resultado dessa seção.

Teorema 24. Seja $f: I \times{ }_{\rho} N^{n-1} \rightarrow \mathbb{Q}_{c}^{n+2}$ uma imersão isométrica, com $n \geq 3$. As seguintes afirmativas são verdadeiras:

(i) se $c \neq 0$ então a segunda forma fundamental de $f$ é adaptada a rede produto de $I \times{ }_{\rho} N^{n-1}$;

(ii) se $c=0$ e $N^{n-1}$ não é flat, então a segunda forma fundamental de $f$ é adaptada a rede produto de $I \times{ }_{\rho} N^{n-1}$.

Demonstração. Se $I \times{ }_{\rho} N^{n-1}$ é livre de pontos com curvatura seccional constante igual a c então, em virtude da Proposição 16 e (DAJCZER; TOJEIRO, 2004, Proposition 36), segue que $f$ não pode ser do tipo B ou C, logo $f$ é do tipo A e, portanto, o resultado segue. Suponhamos agora que $I \times{ }_{\rho} N^{n-1}$ tenha pontos com curvatura seccional constante igual a $c$. Suponhamos ainda que exista um aberto $D_{q}=I_{q} \times{ }_{\rho} N^{n-1} \subset I \times{ }_{\rho} N^{n-1}$ tal que $D_{q}$ tenha curvatura seccional constante igual a $c$, e mostremos que $f$ é tipo A em todos os pontos de $D_{q}$. De fato, pelo Teorema 12 , em cada ponto $z \in D_{q}$, tem-se que ou $R^{\perp}=0$, ou $v_{f}=n-2$. Analisemos cada um desses dois casos.

Caso 1: Suponha que exista um ponto $z \in D_{q}$ tal que $R^{\perp}(z)=0$. Então, pela Proposição 3, existe uma base ortonormal $\left\{X_{1}, X_{2}, \ldots, X_{n}\right\}$ de $T_{z}\left(L \times \rho N^{n-1}\right)$ tal que

$$
\alpha_{f}\left(X_{i}, X_{j}\right)=0
$$

para quaisquer $1 \leq i \neq j \leq n$. Seja

$$
\left\{X, V_{1}, \ldots, V_{n-1}\right\} \subset T_{z}\left(L \times_{\rho} N^{n-1}\right)
$$

uma base ortonormal em relação à decomposição $\mathscr{H}_{z} \oplus \mathscr{V}_{z}$, i.e., $X \in \mathscr{H}_{z}$ e $V_{i} \in \mathscr{V}_{z}$, para todo $1 \leq i \leq n-1$. Se $X=X_{i}$, para algum $1 \leq i \leq n$, e $V_{j}=X_{j}$ para os demais índices $1 \leq i \neq j \leq n$, segue que $f$ é tipo A em $z$. Caso contrário, por (2.4), podemos supor que $\operatorname{dim} E_{1}(z)=1, \operatorname{logo}$

$$
T_{z}\left(I \times{ }_{\rho} N^{n-1}\right)=E_{1}(z) \oplus E_{2}^{n-1}(z) .
$$

O objetivo agora é mostrar que $\mathscr{H}_{z}=E_{1}(z)$ e $\mathscr{V}_{z}=E_{2}^{n-1}(z)$, de onde obteremos (3.19). Suponha, por absurdo, que $X=a_{1} X_{1}+a_{2} E$, onde $a_{1}, a_{2}$ são constantes não-nulas e $E \in E_{2}(z)$, e que exista 
algum índice $1 \leq k \leq n-1$ tal que $V_{k}=b_{1} X_{1}+b_{2} F$, onde $b_{1}, b_{2}$ são constantes não-nulas e $F \in E_{2}(z)$. Temos que

$$
\begin{aligned}
\alpha_{f}\left(X, V_{k}\right) & =\alpha_{f}\left(a_{1} X_{1}+a_{2} E, b_{1} X_{1}+b_{2} F\right)=\alpha_{f}\left(a_{1} X_{1}, b_{1} X_{1}\right) \\
& =a_{1} b_{1}\left\langle X_{1}, X_{1}\right\rangle \eta_{1}=a_{1} b_{1} \eta_{1} \neq 0 .
\end{aligned}
$$

Portanto, $f$ é tipo B no ponto $z$ e, pelo Lema 15 , tem-se

$$
\left\{V_{1}, \ldots, V_{k-1}, V_{k+1}, \ldots, V_{n-1}\right\} \subset \Delta(z)=E_{2}^{n-1} \text {. }
$$

Sem perda de generalidade, podemos considerar $\left\{V_{1}, \ldots, V_{k-1}, V_{k+1}, \ldots, V_{n-1}, F\right\}$ uma base ortonormal para $E_{2}^{n-1}$. Como $F \perp V_{i}$, para todo $1 \leq i \neq k \leq n-1$, então em relação à decomposição $T_{z}\left(I \times_{\rho} N^{n-1}\right)=\mathscr{H}_{z} \oplus \mathscr{V}_{z}$ apenas três possibilidades poderão ocorrer: ou $F \in$ $\mathscr{H}_{z}$, daí $\left\langle b_{1} X_{1}+b_{2} F, F\right\rangle=\left\langle V_{k}, F\right\rangle=0$, contradizendo o fato que $b_{2} \neq 0$; ou $F \in \mathscr{V}_{z}$, daí $\left\langle a_{1} X_{1}+a_{2} E, F\right\rangle=\langle X, F\rangle=0$, o que contradiz o fato que $a_{2} \neq 0$; ou $F=c_{1} X+c_{2} V_{k}$, com $c_{1}, c_{2}$ nã-nulos. Neste caso, $X=a_{1} X_{1}+a_{2} F=a_{1} X_{1}+a_{2} c_{1} X+a_{2} c_{2} V_{k}$, o que nos diz que $a_{1}=a_{2}=0$ e, novamente, teremos uma contradição. Logo, $X \in E_{1}$ e $V_{i} \in E_{2}^{n-1}$ para todo $1 \leq i \leq n-1$. Assim, (3.19) é satisfeita e, portanto, $f$ é tipo A em $z$.

Caso 2: Suponha que exista um ponto $z \in D_{q}$ tal que $v_{f}=n-2$. Assim, $v_{f}=n-2 \mathrm{em}$ uma vizinhança de $z$, a qual vamos supor que seja $D_{q}$. Suponhamos que $f$ seja tipo C em algum ponto $z \in D_{q}$. Assim, pela Proposição 15 , temos que

$$
\operatorname{ker} B_{X}=\operatorname{span}\left\{e_{1}, \ldots, e_{n-3}\right\}
$$

Se existe algum $E \in T_{z} D_{q}$ tal que $\alpha(E, F)=0$, para todo $F \in T_{z} D_{q}$, então $E=X_{0}+V_{0}$, onde $X_{0} \in \mathscr{H}_{z}$ e $V_{0} \in \mathscr{V}_{z} \backslash \operatorname{ker} B_{X}$. Como

$$
\left\langle\alpha\left(E_{1}, E_{2}\right), \alpha\left(E_{3}, E_{4}\right)\right\rangle-\left\langle\alpha\left(E_{1}, E_{4}\right), \alpha\left(E_{2}, E_{3}\right)\right\rangle=0
$$

para quaiquer $E_{1}, E_{2}, E_{3}, E_{4} \in T_{z} D_{q}$, então pelo Teorema 7 , podemos escolher $V_{1} \in\left(\operatorname{ker} B_{X_{0}}\right)^{\perp}$ tal que $\left\langle\alpha\left(V_{0}, V_{0}\right), \alpha\left(V_{1}, V_{1}\right)\right\rangle=0$ e, assim, $\alpha\left(V_{0}, V_{1}\right)=0$. Segue-se então que

$$
0=\alpha\left(E, V_{1}\right)=\alpha\left(X_{0}, V_{1}\right)+\alpha\left(V_{0}, V_{1}\right)=\alpha\left(X_{0}, V_{1}\right)
$$

Isso implica que $V_{1} \in \operatorname{ker} \alpha\left(X, \mathscr{V}_{z}\right)$, o que é uma contradição. Assim, $v_{f}(z)=n-3$, contradizendo a minimalidade de $f$, que é $v_{f}=n-2$. Portanto, $f$ não pode ser tipo $C$ no ponto $z$. Suponhamos agora que $f$ seja do tipo B em $D_{q}$. Assim, de acordo com a Proposição 17, temos que $f$ é do tipo $B_{1}$ ou do tipo $B_{2}$ em $D_{q}$. Consideremos, separadamente, estes dois subcasos.

Subcaso 1: Se $f$ é do tipo $B_{1}$ em $D_{q}$ então, segue de (DAJCZER; TOJEIRO, 2004, Proposition 27) que, localmente, $f$ é uma composição $\left.f\right|_{D_{q}}=F \circ H$, onde $F$ é o produto warped de imersões isométricas $F=\psi \circ\left(f_{1} \times f_{2}\right)$, determinada por uma representação de produto warped $\psi$ : $V^{k_{1}+1} \times_{\sigma} \mathbb{Q}_{\tilde{c}}^{n+k_{2}-1} \rightarrow \mathbb{Q}_{c}^{n+1}$, com $k_{1}+k_{2}=1$, e $H: W \rightarrow \mathbb{Q}_{c}^{n+2}$ é uma imersão isométrica de 
um subconjunto aberto $W$ de $\mathbb{Q}_{c}^{n+1}, \operatorname{com} F\left(D_{q}\right) \subset W$. Considerando $k_{1}=1$ e $k_{2}=0$, segue da Proposição 2 que $v_{F} \geq n-1, \log v_{f} \geq n-1$, o que é uma contradição. Se $k_{1}=0$ e $k_{2}=1$ então, analogamente ao caso em que $k_{1}=1$ e $k_{2}=0$, obtemos uma contradição.

Subcaso 2: Se $f$ for do tipo $B_{2}$ em $D_{q}$ então, como $\tilde{\delta}_{0}=0$, onde $\tilde{\delta}_{0}$ é dado pela Proposição 18 , segue de (DAJCZER; TOJEIRO, 2004, Corollary 33) que o espaço ambiente não pode ser a esfera. Assim, localmente, temos que $N^{n-1}=J \times N_{0}^{n-2}$ é um produto Riemanniano, onde $J \subset \mathbb{R}$ é um intervalo aberto, e existe uma isometria local $i_{2}: N_{0}^{n-2} \rightarrow \mathbb{R}^{n-2}$ e uma imersão isométrica $h: I \times J \rightarrow \mathbb{R}^{4}$ tal que $f=h \times i_{2}$. Segue-se então que $L \times{ }_{\rho} N^{n-1}$ é um produto Riemanniano, logo segue de (DAJCZER; TOJEIRO, 2004, Corollary 32) que o ambiente não pode ser o espaço hiperbólico. Portanto, o ambiente deve ser o espaço Euclidiano, de onde obtemos o item $(i)$.

Quanto ao item (ii), por (DAJCZER; TOJEIRO, 2004, Corollary 33) obtemos que $N^{n-1}$ é flat, o que é uma contradição. Portanto, $f$ é do tipo A em todo $D_{q}, \operatorname{logo} f$ tem a segunda forma fundamental adaptada à rede produto de $I \times{ }_{\rho} N^{n-1}$. Isso prova que $f\left(I \times{ }_{\rho} N^{n-1}\right)$ é um produto warped de imersões isométricas.

\subsection{Subvariedades Euclidianas de codimensão 2}

Nesta seção apresentaremos uma classificação das subvariedades Euclidianas de codimensão 2, sob a ação de um grupo de Lie, com cohomogeneidade 1 e cujas órbitas principais são hipersuperfícies umbílicas. Consideraremos separadamente os casos em que $M$ é compacta e completa.

\subsubsection{O caso compacto}

Abordaremos, inicialmente, o caso em que a subvariedade $M$ é compacta, e mostraremos que $f(M)$ será ou uma subvariedade rotacional ou um produto warped de hipersuperfícies.

Teorema 25. Seja $f: M^{n} \rightarrow \mathbb{R}^{n+2}$ uma imersão isométrica, com $M^{n}$ compacta e $n \geq 4$, e considere um subgrupo conexo e fechado $G \subset I$ so $\left(M^{n}\right)$ agindo sobre $M^{n}$ com cohomogeneidade 1 de modo que as órbitas principais são hipersuperfícies umbílicas de $M^{n}$. Então, $f\left(M^{n}\right)$ é uma subvariedade rotacional ou um produto warped de hipersuperfícies.

Demonstração. Suponhamos inicialmente que a variedade $M^{n}$ tenha curvatura seccional constante igual a $c$. Como $M^{n}$ é compacta, segue do Teorema 8 que $c>0$. Dado um ponto regular $p \in M^{n}$, seja $\Sigma$ uma seção e seja $L$ uma componente conexa da interseção $\Sigma \cap M_{\text {reg. }}$. Considerando o produto warped $L \times{ }_{\rho} G(p)^{n-1}$, com $p \in L$, segue do Teorema 20 que a aplicação

$$
\psi: L \times{ }_{\rho} G(p)^{n-1} \rightarrow M_{r e g}
$$

dada por $\psi(x, g(p))=g(x)$, é uma isometria local. Consideramos então a imersão isométrica

$$
\bar{f}: L \times \rho G(p)^{n-1} \rightarrow \mathbb{R}^{n+2}
$$


dada por $\bar{f}=f \circ \psi$. Por hipótese, temos que o produto warped $L \times_{\rho} G(p)^{n-1}$ é livre de pontos com curvatura seccional constante igual a zero. Como $L$ é unidimensional, segue da Proposição 16 que $\bar{f}$ é do tipo $A$ em todo $L \times_{\rho} G(p)^{n-1}$. Portanto, $\bar{f}$ possui segunda forma fundamental $\alpha_{\bar{f}}$ adaptada à rede produto de $L \times G(p)^{n-1}$.

Suponhamos agora que a variedade $M^{n}$ tenha algum ponto com curvatura seccional constante igual a zero. Em particular, podemos supor que $L \times_{\rho} G(p)^{n-1}$ tenha pontos com curvatura seccional constante igual a zero e pelo Teorema 24 , segue que $\bar{f}$ tem segunda forma fundamental adaptada à rede produto de $L \times{ }_{\rho} G(p)^{n-1}$. Logo pelo teorema de decomposição de Nölker (NÖLKER, 1996), $\bar{f}\left(L \times \rho G(p)^{n-1}\right)$ satisfaz uma das seguintes condições:

1. $\bar{f}\left(L \times{ }_{\rho} G(p)^{n-1}\right)$ é um produto warped de uma imersão isométrica $f_{1}: L \rightarrow V^{3}$ com uma isometria $f_{2}: G(p)^{n-1} \rightarrow \mathbb{S}_{c}^{n-1}, c>0$, determinada por uma representação produto warped $\phi: V^{3} \times_{\bar{\rho}} \mathbb{S}_{c}^{n-1} \rightarrow \mathbb{R}^{n+2} ;$

2. $\bar{f}\left(L \times{ }_{\rho} G(p)^{n-1}\right)$ é um produto warped de duas imersões isométricas $f_{1}: L \rightarrow V^{2}$ e $f_{2}: G(p)^{n-1} \rightarrow \mathbb{S}_{c}^{n}, c>0$, determinada por uma representação produto warped $\phi: V^{2} \times \bar{\rho}$ $\mathbb{S}_{c}^{n} \rightarrow \mathbb{R}^{n+2}$

3. $\bar{f}\left(L \times{ }_{\rho} G(p)^{n-1}\right)$ é o produto warped de uma isometria local $f_{1}: L \rightarrow V$ com uma imersão isométrica $f_{2}: G(p)^{n-1} \rightarrow \mathbb{S}_{c}^{n+1}, c>0$, determinada por uma representação de produto warped $\phi: V \times \bar{\rho}_{c} \mathbb{S}_{c}^{n+1} \rightarrow \mathbb{R}^{n+2}$.

Analisaremos a imersão isométrica $f$ considerando os casos acima. Note que, pela definição de $\bar{f}$, segue que para quaisquer $x \in L$ e $g \in G$, temos que

$$
\bar{f}(x, g(p))=f(\psi(x, g(p)))=f(g(x)) .
$$

Assim, pela Proposição 4, obtemos

$$
f\left(M_{\text {reg }}\right)=f(G(L))=\bar{f}(L, G(p)) .
$$

Disso decorre que

$$
\bar{f}(x, g(p))=\phi\left(f_{1}(x), f_{2}(g(p))\right),
$$

para quaisquer $x \in L$ e $g \in G$. Assim,

$$
f\left(M_{\text {reg }}\right)=\bar{f}(L, G(p))=\phi\left(f_{1}(L), f_{2}(G(p))\right) .
$$

Portanto, pela densidade de $M_{\text {reg }}$ em $M^{n}$ segue que $f\left(M^{n}\right)$ satisfaz uma das três afirmativas acima.

No terceiro caso, obtemos que $f\left(M^{n}\right)$ é o produto warped de uma isometria local $f_{1}: L \rightarrow V$ com uma imersão isométrica $f_{2}: G(p)^{n-1} \rightarrow \mathbb{S}_{c}^{n+1}$, com $c>0$, determinada por uma representação de produto warped $\phi: V \times \bar{\rho}_{\bar{\rho}} \mathbb{S}_{c}^{n+1} \rightarrow \mathbb{R}^{n+2}$. Assim, $f\left(M^{n}\right)$ é um cone compacto sobre a subvariedade $f_{2}$, e logo completo. Como não existem cones completos sobre subvariedades de esferas, obtemos uma contradição. Isso, portanto, finaliza a prova do teorema. 


\subsubsection{O caso completo}

Nesta seção abordaremos o caso em que $M^{n}$ é uma variedade Riemanniana completa, não-compacta. Se o subgrupo $G \subset I s o(M)$ for compacto, mostraremos que a subvariedade $f(M)$ é rotacional, ou um produto warped de hipersuperfícies ou um $(n-2)$-cilindro. Caso o subgrupo $G$ não seja compacto, mostraremos que a subvariedade $f(M)$ é um produto warped de hipersuperfícies ou um $(n-2)$-cilindro.

Teorema 26. Seja $f: M^{n} \rightarrow \mathbb{R}^{n+2}$ uma imersão isométrica, com $M^{n}$ completa, não-compacta e $n \geq 4$, e considere um subgrupo conexo e fechado $G \subset I s o\left(M^{n}\right)$ agindo sobre $M^{n}$ com cohomogeneidade 1 de modo que as órbitas principais são hipersuperfícies umbílicas de $M^{n}$.

1. Se $G$ é compacto, então $f(M)$ satifaz uma das seguintes possibilidades:

a) $f(M)$ é uma subvariedade rotacional,

b) $f(M)$ é um produto warped de hipersuperfícies,

c) $f(M)$ é um $(n-2)$-cilindro.

2. Se $G$ não é compacto, então $f(M)$ satisfaz uma das seguintes possibilidades:

a) $f(M)$ é um produto warped de hipersuperfíces,

b) $f(M)$ é um $(n-2)$-cilindro.

Demonstração. Suponhamos inicialmente que a variedade $M^{n}$ tenha curvatura seccional constante igual a $c$. Se $c=0$, então pelo Teorema 9 , temos $f(M)$ é um $(n-2)$-cilindro. Se $c \neq 0$, então $M^{n}$ é livre de pontos com curvatura seccional constante igual a zero. Dado um ponto regular $p \in M^{n}$, seja $\Sigma$ uma seção de $M$ e seja $L$ uma componente conexa da interseção $\Sigma \cap M_{\text {reg }}$. Considere a imersão isométrica

$$
\bar{f}: L \times{ }_{\rho} G(p)^{n-1} \rightarrow \mathbb{R}^{n+2}
$$

dada por $\bar{f}=f \circ \psi$, onde $\psi$ é a isometria local dada pelo Teorema 20. Suponhamos que a variedade $M^{n}$ seja livre de pontos com curvatura seccional constante igual zero. Em particular, o produto warped $L \times{ }_{\rho} G(p)^{n-1}$ é livre de pontos com curvatura seccional constante igual a zero. Como $L$ é unidimensional, segue pela Proposição 16 que $\bar{f}$ é do tipo $A$ em todo $L \times{ }_{\rho} G(p)^{n-1}$. Portanto, $\bar{f}$ possui a segunda forma fundamental $\alpha_{\bar{f}}$ adaptada à rede produto de $L \times G(p)^{n-1}$.

Suponhamos agora que a variedade $M^{n}$ tenha algum ponto com curvatura seccional constante igual a zero. Em particular, podemos supor que $L \times_{\rho} G(p)^{n-1}$ tenha pontos com curvatura seccional constante igual a zero. Assim, pela Proposição 24, segue que $\bar{f}$ tem a segunda forma fundamental adaptada à rede produto de $L \times{ }_{\rho} G(p)^{n-1}$. Logo, pelo teorema de decomposição de Nölker (NÖLKER, 1996), $\bar{f}\left(L \times \rho G(p)^{n-1}\right)$ satisfaz uma das seguintes possibilidades: 
1. $\bar{f}\left(L \times \rho G(p)^{n-1}\right)$ é um produto warped de uma imersão isométrica $f_{1}: L \rightarrow V^{3}$ com uma isometria $f_{2}: G(p)^{n-1} \rightarrow \mathbb{S}_{c}^{n-1}, c>0$, determinada por uma representação de produto warped $\phi: V^{3} \times_{\bar{\rho}} \mathbb{S}_{c}^{n-1} \rightarrow \mathbb{R}^{n+2}$;

2. $\bar{f}\left(L \times \rho G(p)^{n-1}\right)$ é um produto warped de duas imersões isométricas $f_{1}: L \rightarrow V^{2}$ e $f_{2}: G(p)^{n-1} \rightarrow \mathbb{S}_{c}^{n}, c>0$, determinada por uma representação de produto warped $\phi:$ $V^{2} \times \mathbb{\rho}_{c}^{n} \rightarrow \mathbb{R}^{n+2}$

3. $\bar{f}\left(L \times{ }_{\rho} G(p)^{n-1}\right)$ é o produto warped de uma isometria local $f_{1}: L \rightarrow V$ com uma imersão isométrica $f_{2}: G(p)^{n-1} \rightarrow \mathbb{S}_{c}^{n+1}, c>0$, determinada por uma representação de produto warped $\phi: V \times \bar{\rho} \mathbb{S}_{c}^{n+1} \rightarrow \mathbb{R}^{n+2}$.

Analisaremos a imersão isométrica $f$ considerando os três casos acima. Inicialmente, note que para todo $x \in L$ e $g \in G$, por definição de $\bar{f}$ temos que

$$
\bar{f}(x, g(p))=f(\psi(x, g(p)))=f(g(x)) .
$$

Assim, pela Proposição 4, temos

$$
f\left(M_{\text {reg }}\right)=f(G(L))=\bar{f}(L, G(p)),
$$

$\log 0$

$$
\bar{f}(x, g(p))=\phi\left(f_{1}(x), f_{2}(g(p))\right),
$$

para quaisquer $x \in L$ e $g \in G$. Assim,

$$
f\left(M_{\text {reg }}\right)=\bar{f}(L, G(p))=\phi\left(f_{1}(L), f_{2}(G(p))\right) .
$$

Como $M_{\text {reg }}$ é denso em $M^{n}$ segue que $f\left(M^{n}\right)$ fica completamente determinado por uma das afirmativas acima. Se o subgrupo $G$ é compacto, note que o terceiro caso não pode ocorrer, pois caso ocorra, então teremos que $\bar{f}$ é um cone, $\log f$ será um cone completo sobre $\mathbb{S}_{c}^{n+1}$, o que é uma contradição. Portanto, obtemos o resultado desejado.

Se o subgrupo $G$ não é compacto, então no primeiro caso, $\bar{f}\left(L \times_{\rho} G(p)^{n-1}\right)$ é uma subvariedade rotacional. Porém, como $G(p)^{n-1}$ não é compacto, não podemos ter uma isometria $f_{2}: G(p)^{n-1} \rightarrow \mathbb{S}_{c}^{n-1}$. Assim, este caso não pode ocorrer.

No segundo caso, temos que $\bar{f}(x, g(p))=\phi\left(f_{1}(x), f_{2}(g(p))\right)$ para quaisquer $x \in L$ e $g \in G$. Assim,

$$
f\left(M_{\text {reg }}\right)=\bar{f}(L, G(p))=\phi\left(f_{1}(L), f_{2}(G(p))\right),
$$

$\operatorname{logo} f\left(M^{n}\right)$ é um produto warped de duas imersões isométricas $f_{1}: L \rightarrow V^{2}$ e $f_{2}: G(p)^{n-1} \rightarrow \mathbb{S}_{c}^{n}$, com $c>0$, determinada por uma representação de produto warped $\phi: V^{2} \times_{\bar{\rho}} \mathbb{S}_{c}^{n} \rightarrow \mathbb{R}^{n+2}$.

No terceiro caso, obtemos que $f\left(M^{n}\right)$ é o produto warped de uma isometria local $f_{1}: L \rightarrow V$ com uma imersão isométrica $f_{2}: G(p)^{n-1} \rightarrow \mathbb{S}_{c}^{n+1}, c>0$, determinada por uma representação produto warped $\phi: V \times_{\bar{\rho}} G(p)^{n-1} \rightarrow \mathbb{R}^{n+2}$. Porém, novamente teremos que $\bar{f}$ será um cone, logo $f$ será um cone completo e assim, obtemos uma contradição. 


\subsection{Subvariedades de codimensão 2 na esfera}

Nesta seção apresentaremos uma classificação das subvariedades de codimensão 2 na esfera $\mathbb{S}^{n+2}$ sob a ação de um grupo de Lie, com cohomogeneidade 1 e cujas órbitas principais são hipersuperfícies umbílicas. Como no caso Euclidiano, consideraremos separadamente os casos em que $M$ é compacta e completa.

\subsubsection{O caso compacto}

Teorema 27. Seja $f: M^{n} \rightarrow \mathbb{S}^{n+2}$ uma imersão isométrica, com $M^{n}$ compacta e $n \geq 4$, e considere um subgrupo conexo e fechado $G \subset I s o\left(M^{n}\right)$ agindo sobre $M^{n}$ com cohomogeneidade 1 de modo que as órbitas principais são hipersuperfícies umbílicas de $M^{n}$. Então, $f(M)$ é uma subvariedade rotacional ou um produto warped de hipersuperfícies ou uma esfera.

Demonstração. Suponhamos inicialmente que a variedade $M^{n}$ tenha curvatura seccional constante igual a $c$. Se $c=1$, então pelo Teorema 13, segue que $f$ é totalmente geodésica, logo $f(M)$ é uma esfera. Se $c \neq 1$, então $M$ é livre de pontos com curvatura seccional constante igual a 1 .

Dado um ponto regular $p \in M^{n}$, seja $\Sigma$ uma seção e seja $L$ uma componente conexa da interseção $\Sigma \cap M_{\text {reg. }}$. Considere a imersão isométrica

$$
\bar{f}: L \times{ }_{\rho} G(p)^{n-1} \rightarrow \mathbb{S}^{n+2}
$$

dada por $\bar{f}=f \circ \psi$, onde $\psi$ é a isometria local dada pelo Teorema 20.

Suponhamos que a variedade $M^{n}$ seja livre de pontos com curvatura seccional constante igual 1. Em particular, o produto warped $L \times{ }_{\rho} G(p)^{n-1}$ é livre de pontos com curvatura seccional constante igual a 1 . Como $L$ é unidimensional, segue pela Proposição 16 que $\bar{f}$ é do tipo $A$ em todo $L \times_{\rho} G(p)^{n-1}$. Portanto, $\bar{f}$ possui segunda forma fundamental $\alpha_{\bar{f}}$ adaptada à rede produto de $L \times G(p)^{n-1}$.

Suponhamos agora que a variedade $M^{n}$ tenha algum ponto com curvatura seccional constante igual a 1 . Em particular, podemos supor que $L \times{ }_{\rho} G(p)^{n-1}$ tenha pontos com curvatura seccional constante igual a 1 . Assim, pela Proposição 24 , segue que $\bar{f}$ tem segunda forma fundamental adaptada à rede produto de $L \times_{\rho} G(p)^{n-1}$. Logo pelo teorema de decomposição de Nölker (NÖLKER, 1996), segue que $\bar{f}\left(L \times{ }_{\rho} G(p)^{n-1}\right)$ satisfaz uma das seguintes possibilidades:

1. $\bar{f}\left(L \times{ }_{\rho} G(p)^{n-1}\right)$ é um produto warped de uma imersão isométrica $f_{1}: L \rightarrow V^{3}$ com uma isometria $f_{2}: G(p)^{n-1} \rightarrow \mathbb{S}_{c}^{n-1}, c>0$, determinada por uma representação produto warped $\phi: V^{3} \times \bar{\rho} \mathbb{S}_{c}^{n-1} \rightarrow \mathbb{S}^{n+2}$

2. $\bar{f}\left(L \times{ }_{\rho} G(p)^{n-1}\right)$ é um produto warped de duas imersões isométricas $f_{1}: L \rightarrow V^{2} \mathrm{e}$ $f_{2}: G(p)^{n-1} \rightarrow \mathbb{S}_{c}^{n}, c>0$, determinada por uma representação produto warped $\phi: V^{2} \times \bar{\rho}$ $\mathbb{S}_{c}^{n} \rightarrow \mathbb{S}^{n+2}$ 
3. $\bar{f}\left(L \times{ }_{\rho} G(p)^{n-1}\right)$ é o produto warped de uma isometria local $f_{1}: L \rightarrow V$ com uma imersão isométrica $f_{2}: G(p)^{n-1} \rightarrow \mathbb{S}_{c}^{n+1}, c>0$, determinada por uma representação de produto warped $\phi: V \times \bar{\rho} \mathbb{S}_{c}^{n+1} \rightarrow \mathbb{S}^{n+2}$.

Analisaremos a imersão isométrica $f$ considerando os três casos acima. Inicialmente, note que para quaisquer $x \in L$ e $g \in G$, por definição de $\bar{f}$ temos que

$$
\bar{f}(x, g(p))=f(\psi(x, g(p)))=f(g(x)) .
$$

Assim, pela Proposição 4, temos

$$
f\left(M_{\text {reg }}\right)=f(G(L))=\bar{f}(L, G(p)),
$$

$\log 0$

$$
\bar{f}(x, g(p))=\phi\left(f_{1}(x), f_{2}(g(p))\right),
$$

para quaisquer $x \in L$ e $g \in G$. Assim,

$$
f\left(M_{\text {reg }}\right)=\bar{f}(L, G(p))=\phi\left(f_{1}(L), f_{2}(G(p))\right) .
$$

Como $M_{\text {reg }}$ é denso em $M^{n}$ segue que a subvariedade $f(M)$ satisfaz um dos três casos acima. Daremos atenção especial para o terceiro caso, pois neste caso, $f\left(M^{n}\right)$ será um cone completo sobre $\mathbb{S}_{c}^{n+1}$, como não existe cone completo sobre subvariedades de esferas em $\mathbb{S}^{n+2}$, obtemos uma contradição. Portanto este caso não ocorre e assim obtemos resultado desejado.

\subsubsection{O caso completo}

Nesta seção abordaremos o caso em que $M^{n}$ é uma variedade Riemanniana completa e não-compacta.

Teorema 28. Seja $f: M^{n} \rightarrow \mathbb{S}^{n+2}$ uma imersão isométrica, com $M^{n}$ completa, não-compacta e $n \geq 4$, e considere um subgrupo conexo e fechado $G \subset I$ so $\left(M^{n}\right)$ agindo sobre $M^{n}$ com cohomogeneidade 1 de modo que as órbitas principais são hipersuperfícies umbílicas de $M^{n}$.

1. Se $G$ é compacto, então $f(M)$ satisfaz uma das seguintes possibilidades:

a) $f(M)$ é uma subvariedade rotacional;

b) $f(M)$ é um produto warped de hipersuperfícies;

c) $f(M)$ é uma esfera.

2. Se $G$ não é compacto, então $f(M)$ satisfaz uma das seguintes possibilidades:

a) $f(M)$ é produto warped de hipersuperfícies,

b) $f(M)$ é uma esfera. 
Demonstração. Suponhamos inicialmente que a variedade $M^{n}$ tenha pontos com curvatura seccional constante igual $c$. Se $c=1$, então pelo Teorema 13, segue que $f$ é totalmente geodésica, $\operatorname{logo} f(M)$ é uma esfera. Se $c \neq 1$, então $M^{n}$ é livre de pontos com curvatura seccional constante igual a 1 .

Dado um ponto regular $p \in M^{n}$, seja $\Sigma$ uma seção de $M$ e seja $L$ uma componente conexa da interseção $\Sigma \cap M_{\text {reg. }}$. Considere a imersão isométrica

$$
\bar{f}: L \times{ }_{\rho} G(p)^{n-1} \rightarrow \mathbb{R}^{n+2}
$$

dada por $\bar{f}=f \circ \psi$, onde $\psi$ é a isometria local dada pelo Teorema 20. Suponhamos que a variedade $M^{n}$ seja livre de pontos com curvatura seccional constante igual 1. Em particular, o produto warped $L \times{ }_{\rho} G(p)^{n-1}$ é livre de pontos com curvatura seccional constante igual a 1 . Como $L$ é unidimensional, segue da Proposição 16 que $\bar{f}$ é do tipo $A$ em todo $L \times{ }_{\rho} G(p)^{n-1}$. Portanto, $\bar{f}$ possui a segunda forma fundamental $\alpha_{\bar{f}}$ adaptada à rede produto de $L \times G(p)^{n-1}$.

Suponhamos agora que a variedade $M^{n}$ tenha algum ponto com curvatura seccional constante igual a 1. Em particular, podemos supor que $L \times{ }_{\rho} G(p)^{n-1}$ tenha pontos com curvatura seccional constante igual a 1. Assim, pela Proposição 24, segue que $\bar{f}$ tem a segunda forma fundamental adaptada à rede produto de $L \times \rho G(p)^{n-1}$. Logo, pelo teorema de decomposição de Nölker (NÖLKER, 1996), segue que $\bar{f}\left(L \times{ }_{\rho} G(p)^{n-1}\right)$ satisfaz uma das seguintes possibilidades:

1. $\bar{f}\left(L \times \rho G(p)^{n-1}\right)$ é um produto warped de uma imersão isométrica $f_{1}: L \rightarrow V^{3}$ com uma isometria $f_{2}: G(p)^{n-1} \rightarrow \mathbb{S}_{c}^{n-1}, c>0$, determinada por uma representação produto warped $\phi: V^{3} \times \mathbb{\rho}_{c}^{n-1} \rightarrow \mathbb{S}^{n+2}$

2. $\bar{f}\left(L \times_{\rho} G(p)^{n-1}\right)$ é um produto warped de duas imersões isométricas $f_{1}: L \rightarrow V^{2}$ e $f_{2}: G(p)^{n-1} \rightarrow \mathbb{S}_{c}^{n}, c>0$, determinada por uma representação produto warped $\phi: V^{2} \times \bar{\rho}$ $\mathbb{S}_{c}^{n} \rightarrow \mathbb{S}^{n+2}$

3. $\bar{f}\left(L \times \rho G(p)^{n-1}\right)$ é o produto warped de uma isometria local $f_{1}: L \rightarrow V$ com uma imersão isométrica $f_{2}: G(p)^{n-1} \rightarrow \mathbb{S}_{c}^{n+1}, c>0$, determinada por uma representação de produto warped $\phi: V \times \bar{\rho} \mathbb{S}_{c}^{n+1} \rightarrow \mathbb{S}^{n+2}$.

Note que para todo $x \in L$ e $g \in G$, por definição de $\bar{f}$ temos que

$$
\bar{f}(x, g(p))=f(\psi(x, g(p)))=f(g(x)) .
$$

Assim, pela Proposição 4, temos

$$
f\left(M_{\text {reg }}\right)=f(G(L))=\bar{f}(L, G(p))
$$

$\log 0$

$$
\bar{f}(x, g(p))=\phi\left(f_{1}(x), f_{2}(g(p))\right),
$$


para quaisquer $x \in L$ e $g \in G$. Assim,

$$
f\left(M_{\text {reg }}\right)=\bar{f}(L, G(p))=\phi\left(f_{1}(L), f_{2}(G(p))\right) .
$$

Como $M_{\text {reg }}$ é denso em $M^{n}$ segue que a subvariedade $f(M)$ satisfaz um dos três casos acima. Se o subgrupo $G$ é compacto, então o terceiro caso não pode ocorrer, pois neste caso, $f(M)$ é um cone completo sobre $\mathbb{S}_{c}^{n+1}$, o que é uma contradição.

Se o subgrupo $G$ não é compacto, então no primeiro caso, como $G(p)^{n-1}$ não é compacto, não podemos ter uma isometria $f_{2}: G(p) \rightarrow \mathbb{S}_{c}^{n-1}$. Assim, este caso não pode ocorrer. No segundo caso, temos que

$$
\bar{f}(x, g(p))=\phi\left(f_{1}(x), f_{2}(g(p))\right),
$$

para quaisquer $x \in L$ e $g \in G$. Assim,

$$
f\left(M_{\text {reg }}\right)=\bar{f}(L, G(p))=\phi\left(f_{1}(L), f_{2}(G(p))\right),
$$

logo $f(M)$ é um produto warped de duas imersões isométricas $f_{1}: L \rightarrow V^{2}$ e $f_{2}: G(p)^{n-1} \rightarrow \mathbb{S}_{c}^{n}$, $c>0$, determinada por uma representação de produto warped $\phi: V^{2} \times \mathbb{S}_{c}^{n} \rightarrow \mathbb{S}^{n+2}$.

No terceiro caso, obtemos que $f\left(M^{n}\right)$ é o produto warped de uma isometria local $f_{1}: L \rightarrow V$ com uma imersão isométrica $f_{2}: G(p)^{n-1} \rightarrow \mathbb{S}_{c}^{n+1}, c>0$, determinada por uma representação produto warped $\phi: V \times_{\bar{\rho}} G(p)^{n-1} \rightarrow \mathbb{S}^{n+2}$. Assim, $f(M)$ será um cone completo sobre $\mathbb{S}_{c}^{n+1}$, o que é uma contradição. Logo, este caso também não pode ocorrer, e assim obtemos o desejado.

\subsection{Subvariedades de codimensão 2 no espaço hiperbóli- co}

Nesta seção apresentaremos uma classificação das subvariedades de codimensão 2 no espaço hiperbólico $\mathbb{H}^{n+2}$ sob a ação de um grupo de Lie, com cohomogeneidade 1 e cujas órbitas principais são hipersuperfícies umbílicas. Como nos demais casos, consideraremos separadamente os casos em que $M$ é compacta e completa.

\subsubsection{O caso compacto}

Nesta subseção abordaremos o caso em que a variedade Riemanniana $M^{n}$ é compacta, e mostraremos que $f(M)$ será uma subvariedade rotacional elíptica, ou um produto warped de hipersuperfícies: ou elíptica, ou hiperbólica ou parabólica, ou um cone: parabólico ou hiperbólico.

Teorema 29. Seja $f: M^{n} \rightarrow \mathbb{H}^{n+2}$ uma imersão isométrica, com $M^{n}$ compacta e $n \geq 4$, e considere um subgrupo conexo e fechado $G \subset I s o\left(M^{n}\right)$ agindo sobre $M^{n}$ com cohomogeneidade 1 de modo que as órbitas principais são hipersuperfícies umbílicas de $M^{n}$. Então $f(M)$ satisfaz uma das seguintes possibilidades: 
1. $f(M)$ é uma subvariedade rotacional elíptica;

2. $f(M)$ é um produto warped de hipersuperfícies: elíptico, ou hiperbólico, ou parabólico.

3. $f(M)$ é um cone: hiperbólico ou parabólico.

Demonstração. Suponhamos, inicialmente, que a variedade $M^{n}$ tenha curvatura seccional constante igual a $c$. Assim, pelo Teorema 10 , segue que $c>-1$ e a variedade $M^{n}$ é livre de pontos com curvatura seccional constante igual a -1 .

Dado um ponto regular $p \in M^{n}$, seja $\Sigma$ uma seção e seja $L$ uma componente conexa da interseção $\Sigma \cap M_{\text {reg. }}$. Considere a imersão isométrica

$$
\bar{f}: L \times_{\rho} G(p)^{n-1} \rightarrow \mathbb{H}^{n+2}
$$

dada por $\bar{f}=f \circ \psi$, onde $\psi$ é a isometria local dada pelo Teorema 20.

Suponhamos que a variedade $M^{n}$ seja livre de pontos com curvatura seccional constante igual -1 . Em particular, o produto warped $L \times{ }_{\rho} G(p)^{n-1}$ é livre de pontos com curvatura seccional constante igual a -1 . Como $L$ é unidimensional, segue pela Proposição 16 que $\bar{f}$ é do tipo $A$ em todo $L \times{ }_{\rho} G(p)^{n-1}$. Portanto, $\bar{f}$ possui segunda forma fundamental $\alpha_{\bar{f}}$ adaptada à rede produto de $L \times G(p)^{n-1}$.

Suponhamos agora que a variedade $M^{n}$ tenha algum ponto com curvatura seccional constante igual a -1 . Em particular, podemos supor que $L \times_{\rho} G(p)^{n-1}$ tenha pontos com curvatura seccional constante igual a -1 . Assim, pela Proposição 24 , segue que $\bar{f}$ tem segunda forma fundamental adaptada à rede produto de $L \times{ }_{\rho} G(p)^{n-1}$. Logo pelo teorema de decomposição de Nölker (NÖLKER, 1996), segue que $\bar{f}\left(L \times{ }_{\rho} G(p)^{n-1}\right)$ satisfaz:

$$
\bar{f}=\phi \circ\left(f_{1} \times f_{2}\right)
$$

onde $\phi: V^{k_{1}+1} \times_{\sigma} \mathbb{Q}_{c}^{n+k_{2}-1} \rightarrow \mathbb{H}^{n+2}$ é uma representação de produto warped, com $k_{1}+k_{2}=2$, e $f_{1}: L \rightarrow V^{k_{1}+1}$ e $f_{2}: L \rightarrow \mathbb{Q}_{c}^{n+k_{2}-1}$ são imersões isométricas. Para $k_{1}=0$, não teremos um cone elíptico, pois neste caso $f$ seria um cone completo sobre $\mathbb{S}_{c}^{n+1}$. Como não exitem cones completos sobre subvariedades de esferas em $\mathbb{H}^{n+2}$, obtemos uma contradição.

Para $k_{2}=0$, pela compacidade de $G(x)^{n-1}$, temos que $\mathbb{Q}_{c}^{n-1}=\mathbb{S}_{c}^{n-1}$. Logo, obtemos que $\bar{f}: L \times{ }_{\rho} G(p)^{n-1} \rightarrow \mathbb{H}^{n+2}$ é uma subvariedade rotacional elíptica.

Caso $k_{2}=k_{1}=1$, então $\bar{f}$ será um produto warped de hipersuperfícies: elíptica $(c>0)$, ou hiperbólica $(c<0)$, ou parabólica $(c=0)$. Note que, para quaisquer $x \in L$ e $g \in G$, temos

$$
\bar{f}(x, g(p))=f(\psi(x, g(p)))=f(g(x)) .
$$

Assim, pela Proposição 4, temos que

$$
f\left(M_{\text {reg }}\right)=f(G(L))=\bar{f}(L, G(p)),
$$


$\log 0$

$$
\bar{f}(x, g(p))=\phi\left(f_{1}(x), f_{2}(g(p))\right),
$$

para quaisquer $x \in L$ e $g \in G$. Assim,

$$
f\left(M_{\text {reg }}\right)=\bar{f}(L, G(p))=\phi\left(f_{1}(L), f_{2}(G(p))\right) .
$$

Como $M_{\text {reg }}$ é denso em $M^{n}$ segue que a subvariedade $f(M)$ satisfaz um dos casos acima.

\subsubsection{O caso completo}

Nesta subseção abordaremos o caso em que $M^{n}$ é uma variedade Riemanniana completa e não-compacta.

Teorema 30. Seja $f: M^{n} \rightarrow \mathbb{H}^{n+2}$ uma imersão isométrica, com $M^{n}$ completa, não-compacta e $n \geq 4$, e considere um subgrupo conexo e fechado $G \subset I s o\left(M^{n}\right)$ agindo sobre $M^{n}$ com cohomogeneidade 1 de modo que as órbitas principais são hipersuperfícies umbílicas de $M^{n}$.

1. Se $G$ é compacto, então $f(M)$ satifaz uma das seguintes possibilidades:

a) $f(M)$ é uma subvariedade rotacional elíptica;

b) $f(M)$ é um cone: parabólico, ou hiperbólico;

c) $f(M)$ é um produto warped de hipersuperfícies: elíptica, ou parabólica, ou hiperbólica.

2. Se $G$ não é compacto, então $f(M)$ satisfaz uma das seguintes afirmativas:

a) $f(M)$ é uma subvariedade rotacional: parabólica ou hiperbólica;

b) $f(M)$ é produto warped de hipersuperfícies: elíptica, ou parabólica, ou hiperbólica;

c) $f(M)$ é um cone: parabólico ou hiperbólico.

Demonstração. Inicialmente, suponhamos que a variedade $M^{n}$ tenha curvatura constante $c$. Se $c=-1$, então a parte regular $M_{\text {reg }}$ tem curvatura seccional constante igual a -1 . Caso, $c \neq-1$ então $M^{n}$ é livre de pontos com curvatura seccional constante igual a -1 .

Dado um ponto regular $p \in M^{n}$, seja $\Sigma$ uma seção e seja $L$ uma componente conexa da interseção $\Sigma \cap M_{\text {reg }}$. Considere a imersão isométrica

$$
\bar{f}: L \times_{\rho} G(p)^{n-1} \rightarrow \mathbb{H}^{n+2}
$$

dada por $\bar{f}=f \circ \psi$, onde $\psi$ é a isometria local dada pelo Teorema 20.

Suponhamos que a variedade $M^{n}$ seja livre de pontos com curvatura seccional constante igual -1. Em particular, o produto warped $L \times{ }_{\rho} G(p)^{n-1}$ é livre de pontos com curvatura seccional constante igual a -1 . Como $L$ é unidimensional, segue pela Proposição 16 que $\bar{f}$ é do tipo $A$ em 
todo $L \times{ }_{\rho} G(p)^{n-1}$. Portanto, $\bar{f}$ possui segunda forma fundamental $\alpha_{\bar{f}}$ adaptada à rede produto de $L \times G(p)^{n-1}$.

Suponhamos agora que a variedade $M^{n}$ tenha algum ponto com curvatura seccional constante igual a -1 . Em particular, podemos supor que $L \times_{\rho} G(p)^{n-1}$ tenha pontos com curvatura seccional constante igual a -1 . Assim, pela Proposição 24, segue que $\bar{f}$ tem segunda forma fundamental adaptada à rede produto de $L \times{ }_{\rho} G(p)^{n-1}$. Logo pelo teorema de decomposição de Nölker (NÖLKER, 1996), segue que $\bar{f}\left(L \times{ }_{\rho} G(p)^{n-1}\right)$ satisfaz:

$$
\bar{f}=\phi \circ\left(f_{1}, f_{2}\right)
$$

onde $\phi: V^{k_{1}+1} \times_{\sigma} \mathbb{Q}_{c}^{n+k_{2}-1} \rightarrow \mathbb{H}^{n+2}$, com $k_{1}+k_{2}=2, f_{1}: L \rightarrow V^{k_{1}+1}$ e $f_{2}: L \rightarrow \mathbb{Q}_{c}^{n+k_{2}-1}$ são imersões isométricas.

Suponhamos que o subgrupo $G$ seja compacto. Para $k_{1}=0$, não podemos ter um cone elíptico. Para $k_{2}=0$, pela compacidade de $G(x)^{n-1}$, temos que $\mathbb{Q}_{c}^{n-1}=\mathbb{S}_{c}^{n-1}$. Logo, obteremos que $\bar{f}: L \times{ }_{\rho} G(p)^{n-1} \rightarrow \mathbb{H}^{n+2}$ é uma subvariedade rotacional elíptica. Caso $k_{2}=k_{1}=1$, então $\bar{f}$ será um produto warped de hipersuperfícies: elíptica $(c>0)$, ou hiperbólica $(c<0)$, ou parabólica $(c=0)$.

Se o subgrupo $G$ não é compacto, então para $k_{2}=0$ teremos que $\mathbb{Q}_{c}^{n-1}$ não pode ser a esfera $\mathbb{S}_{c}^{n-1}$, pois $G(p)^{n-1}$ não é compacto e não podemos ter uma isometria $f_{2}: G(p) \rightarrow \mathbb{S}_{c}^{n-1}$. Logo, $\bar{f}$ será uma subvariedade rotacional: hiperbólica, ou parabólica.

Para $k_{1}=0$, excluiremos apenas o cone elíptico. Segue que uma outra possibilidade para $\bar{f}$, será quando $k_{1}=k_{2}=1$. Assim, teremos que $\bar{f}$ será um produto warped de hipersuperfícies: elíptica $(c>0)$, ou hiperbólica $(c<0)$, ou parabólica $(c=0)$. Logo, obtemos o desejado. 
ASPERTI, A. C.; CAPUTI, A. Cohomogeneity one hypersurfaces of the hyperbolic space. Annals of Global Analysis and Geometry, Springer, v. 24, n. 4, p. 351-373, 2003. Citado na página 18.

BERNDT, J.; CONSOLE, S.; OLMOS, C. E. Submanifolds and holonomy. [S.1.]: CRC Press, 2016. v. 21. Citado nas páginas 28 e 31.

CASTRO, H. P. D.; NORONHA, M. H. Homogeneous submanifolds of codimension two. Geometriae Dedicata, Springer, v. 78, n. 1, p. 89-110, 1999. Citado na página 18.

. Homogeneous manifolds in codimension two revisited. Note di Matematica, v. 21, n. 1, p. 49-57, 2002. Citado na página 18.

DAJCZER, M.; ANTONUCCI, M.; OLIVEIRA, G.; LIMA-FILHO, P.; TOJEIRO, R. Submanifolds and isometric immersions. [S.1.]: Mathematics Lecture Series 13, Publish or Perish Inc, 1990. Citado nas páginas 23, 24, 25 e 27.

DAJCZER, M.; TOJEIRO, R. Isometric immersions in codimension two of warped products into space forms. Illinois Journal of Mathematics, University of Illinois at Urbana-Champaign, Department of Mathematics, v. 48, n. 3, p. 711-746, 2004. Citado nas páginas 18, 38, 39, 40, $43,44,46,47,48,49$ e 50.

EJIRI, N. A generalization of minimal cones. Transactions of the American Mathematical Society, v. 276, n. 1, p. 347-360, 1983. Citado na página 35.

ERBACHER, J. Isometric immersions of constant mean curvature and triviality of the normal connection. Nagoya Mathematical Journal, Cambridge University Press, v. 45, p. 139-165, 1972. Citado na página 27.

GONÇALVES, I. M. G-variedades riemannianas como hipersuperfícies de formas espaciais. Universidade Federal de São Carlos, 2006. Citado nas páginas 25, 32 e 36.

HARLE, C. E. Rigidity of hypersurfaces of constant scalar curvature. Journal of Differential Geometry, Lehigh University, v. 5, n. 1-2, p. 85-111, 1971. Citado na página 17.

HARTMAN, P. On isometric immersions in euclidean space of manifolds with non-negative sectional curvatures. Transactions of the American Mathematical Society, JSTOR, v. 115, p. 94-109, 1965. Citado na página 27.

HSIANG, W.-y.; JR, H. B. L. Minimal submanifolds of low cohomogeneity. Journal of Differential Geometry, Lehigh University, v. 5, n. 1-2, p. 1-38, 1971. Citado na página 17.

KOBAYASHI, S. Compact homogeneous hypersurfaces. Transactions of the American Mathematical Society, JSTOR, v. 88, n. 1, p. 137-143, 1958. Citado nas páginas 11, 13 e 17.

LIMA, J. C. A. Hipersuperficies de coomogeneidade um na esfera euclidiana. [sn], 2002. Citado na página 17. 
MERCURI, F.; PODESTÀ, F.; SEIXAS, J. A.; TOJEIRO, R. Cohomogeneity one hypersurfaces of euclidean spaces. Commentarii Mathematici Helvetici, v. 81, n. 2, p. 471-479, 2006. Citado na página 17.

MERCURI, F.; SEIXAS, J. A. P. Hypersurfaces of cohomogeneity one and hypersurfaces of revolution. Differential Geometry and its Applications, Elsevier, v. 20, n. 2, p. 225-239, 2004. Citado na página 17.

MOORE, J. Isometric immersions of space forms in space forms. Pacific Journal of Mathematics, Mathematical Sciences Publishers, v. 40, n. 1, p. 157-166, 1972. Citado nas páginas 26 e 27 .

MOORE, J. D. Isometric immersions of riemannian products. Journal of Differential Geometry, Lehigh University, v. 5, n. 1-2, p. 159-168, 1971. Citado nas páginas 18 e 36.

NAGANO, T.; TAKAHASHI, T. Homogeneous hypersurfaces in euclidean spaces. Journal of the Mathematical Society of Japan, The Mathematical Society of Japan, v. 12, n. 1, p. 1-7, 1960. Citado na página 17.

NÖLKER, S. Isometric immersions of warped products. Differential Geometry and its applications, Elsevier, v. 6, n. 1, p. 1-30, 1996. Citado nas páginas 18, 34, 37, 39, 43, 51, 52, 54, 56, 58 e 60 .

O'NEILL, B. Immersion of manifolds of nonpositive curvature. Proceedings of the American Mathematical Society, JSTOR, v. 11, n. 1, p. 132-134, 1960. Citado nas páginas 27 e 35.

PALAIS, R. S.; TERNG, C.-L. A general theory of canonical forms. Transactions of the American Mathematical Society, v. 300, n. 2, p. 771-789, 1987. Citado nas páginas 28 e 32.

Critical point theory and submanifold geometry. [S.1.]: Springer, 2006. v. 1353. Citado na página 28.

PODESTÀ, F.; SPIRO, A. Cohomogeneity one manifolds and hypersurfaces of the euclidean space. Annals of Global Analysis and Geometry, Springer, v. 13, n. 2, p. 169-184, 1995. Citado nas páginas 17 e 36.

ROS, A. Compact hypersurfaces with constant scalar curvature and a congruence theorem. Journal of Differential Geometry, Lehigh University, v. 27, n. 2, p. 215-220, 1988. Citado na página 17.

STIEL, E. Isometric immersions of manifolds of nonnegative constant sectional curvature. Pacific Journal of Mathematics, Mathematical Sciences Publishers, v. 15, n. 4, p. 1415-1419, 1965. Citado na página 28.

TAKAHASHI, T. Homogeneous hypersurfaces in spaces of constant curvature. Journal of the Mathematical Society of Japan, The Mathematical Society of Japan, v. 22, n. 3, p. 395-410, 1970. Citado na página 17.

An isometric immersion of a homogeneous riemannian manifold of dimension 3 in the hyperbolic space. Journal of the Mathematical Society of Japan, The Mathematical Society of Japan, v. 23, n. 4, p. 649-661, 1971. Citado na página 17.

UCHIDA, F. An orthogonal transformation group of $(8 k-1)$-sphere. Journal of Differential Geometry, Lehigh University, v. 15, n. 4, p. 569-574, 1980. Citado na página 17. 


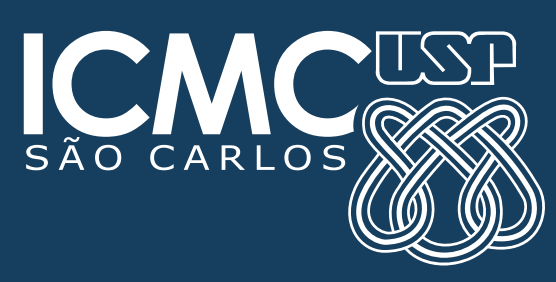

